\title{
A Low-Complexity Cross-Layer Algorithm for Coordinated Downlink Scheduling and Robust Beamforming Under a Limited Feedback Constraint
}

\author{
Yingxiang Yang, Bo Bai, Member, IEEE, Wei Chen, Senior Member, IEEE, and Lajos Hanzo, Fellow, IEEE
}

5 Abstract-Coordinated scheduling/beamforming (CS/CB) sub6 stantially mitigates the intercell interference (ICI), hence increas7 ing the cell-edge throughput on the downlink (DL) of coordinated 8 multipoint (CoMP) systems. To maximize the DL throughput, the 9 cooperating base stations (BSs) jointly select the best set of users 10 for DL scheduling and then jointly design a set of beamforming 11 (BF) vectors to approach the throughput limit. However, finding 12 the optimal BF vectors requires an exhaustive search and sub13 stantial channel state information (CSI) feedback, hence resulting 14 in high algorithmic complexity and heavy uplink traffic load. 15 Hence, we conceive a new cross-layer algorithm to achieve high 16 performance at a lower feedback amount and at lower algorithmic 17 complexity. Based on the fact that different BSs usually have 18 different traffic loads, we divide the BSs into two different types, 19 i.e., the master BSs (MBSs) and the slave BSs (SBSs), where MBSs 20 have a higher transmission priority than SBSs. The scheduling 21 relies on an interference threshold, whereas our robust BF scheme 22 exploits both the channel direction information (CDI), which is 23 quantized using the technique of limited feedback, and the channel 24 quality information (CQI), which is assumed to be fed back accu25 rately. Our numerical results show that the proposed algorithm 26 does not lose much performance compared with that achieved by 27 an exhaustive search, whereas the algorithmic complexity is as low 28 as that of the solutions operating without CoMP.

29 Index Terms-Author, please supply index terms/keywords 30 for your paper. To download the IEEE Taxonomy go to 31 http://www.ieee.org/documents/taxonomy_v101.pdf .

\section{INTRODUCTION}

33

34 OORDINATED multipoint (CoMP) transmission is a key feature in the Long-Term Evolution system [1]-[3], which 35 promises performance improvements for the cell-edge users by 36 allowing several base stations (BSs) to cooperate. On the uplink

Manuscript received August 7, 2012; revised February 5, 2013 and May 17, 2013; accepted June 22, 2013. This paper was supported in part by the National Basic Research Program of China (973 Program) under Grant 2013CB336600 and Grant 2012CB316000, by the U.K.-China Bridge Fellowship, by Chuanxin Funding, by the Beijing Nova Program, and by the Chinese Ministry of Education New Century Talent Program. The work of L. Hanzo was supported by the European Research Council under their Advanced Fellow Grant. The review of this paper was coordinated by Dr. J.-C. Chen.

Y. Yang was with Tsinghua University, Beijing 100084, China. He is currently with the Department of Electrical and Computer Engineering, Rutgers University, New Brunswick, NJ 08901 USA (e-mail: yangyx.thu @ gmail.com).

B. Bai and W. Chen are with the Department of Electronic Engineering, Tsinghua University, Beijing, 100084, China (e-mail:eebobai@tsinghua. edu.cn; wchen@tsinghua.edu.cn).

L. Hanzo is with the School of Electronic and Computer Science, University of Southampton, Southampton, SO17 1BJ U.K. (e-mail: 1h@ecs.soton.ac.uk).

Color versions of one or more of the figures in this paper are available online at http://ieeexplore.iee.org.

Digital Object Identifier 10.1109/TVT.2013.2271876 side, the cooperating BSs share the information and jointly pro- 37 cess the data received from the mobile stations (MSs). On the 38 downlink (DL) side, two commonly implemented methods are 39 the joint processing and coordinated scheduling/beamforming 40 (CS/CB) schemes, where CS/CB allows the BSs to coopera- 41 tively schedule their DL transmissions to a set of users and 42 then cooperatively design a set of efficient beamforming (BF) 43 vectors. Under the assumption that the users' channel state 44 information (CSI) is perfectly known at the BSs' DL trans- 45 mitters, the throughput performance of coordinated BF varies 46 for different sets of scheduled users. Thus, the ultimate task for 47 the BSs is to schedule their DL transmissions to the optimal 48 set of users that are capable of approaching the maximum DL- 49 transmission throughput and then to design the particular set of 50 $\mathrm{BF}$ vectors that can approach this limit.

However, the problem described earlier has the following 52 obstacles.

1) High algorithmic complexity. The algorithmic complex- 54 ity imposed by finding the optimal set of MSs for which 55 the DL transmissions should be scheduled is high since 56 an exhaustive search is required for optimal scheduling. 57 Assuming that there are $M$ BSs and that the user set of 58 the $i$ th $\mathrm{BS}(\mathrm{BS} i)$ is denoted by $\mathcal{U}_{i}$, the complexity of the 59 scheduling algorithm is on the order of $\mathcal{O}\left(\prod_{i=1}^{M}\left|\mathcal{U}_{i}\right|\right) .60$ This complexity becomes excessive, when the number of 61 BSs and MSs in each cell increases.

2) High feedback load. Assuming that the feedback "bud- 63 get" of each MS's CSI is $B$ bits for the Channel Di- 64 rection Information (CDI) and $b$ bits for the Channel 65 Quality Information (CQI), the feedback traffic load can 66 be expressed as $\left(\sum_{i=1}^{M}\left|\mathcal{U}_{i}\right|\right)(M B+b)$, where the $M B 67$ bits of the CDI feedback are related to $M$ channels, i.e., 68 one for the specific channel receiving the desired signal 69 and the remaining $(M-1)$ for the channel receiving the 70 interfering signal.

3) High backhaul traffic. To calculate the set of optimal BF 72 vectors, at least one of the BSs has to know the CSI of 73 all the MSs. Thus, the backhaul traffic load is at least 74 $\left(\sum_{i=1}^{M}\left|\mathcal{U}_{i}\right|-\max _{i}\left\{\left|\mathcal{U}_{i}\right|\right\}\right)(M B+b)$.

4) Inaccuracy caused by imperfect CSI feedback. Since the 76 CSI feedback introduces both quantization errors and 77 latency, the CSI acquired at the BSs is inaccurate. Thus, 78 it is possible that the DL-scheduling decision will be 79 inaccurate when the quantization error is high. 
81 There has been a plethora of contributions related to CoMP 82 [1], [4]-[15], exploring possible solutions and finding remedies 83 to the impediments aforementioned. Although the original con84 tributions relied on the assumption of perfect CSI [4], more 85 realistic recent contributions assumed imperfect CSI feedback, 86 where the channel vectors are quantized to a codeword stored 87 in a codebook designed offline and the index of the code88 word is fed back instead of the actual quantized values of the 89 channel vectors. Hence, the amount of feedback per user can 90 be substantially reduced. A comprehensive introduction to the 91 topic of limited feedback aid communications can be found 92 in [5], where the authors discussed the feedback design in a 93 broad range of scenarios, employing methods used in industrial 94 standards and protocols.

95 One of the common issues in realistic limited-feedback-aided 96 systems is the inaccuracy of the CSI feedback both due to the 97 delay encountered and by the transmission errors imposed by 98 the feedback channels [6]-[10]. In [6], Wu and Lau proposed 99 a feedback design for spatial-division multiple-access (SDMA) 100 systems, demonstrating that their scheme is robust against feed101 back channel errors and characterized the system's goodput. 102 Another contribution of $\mathrm{Wu}$ and Lau [7] provided two robust 103 designs for multiple-input-multiple-output precoder adaptation 104 under the scenario of potentially error-prone limited feedback 105 and showed that both frameworks provided significant gains 106 compared with the idealized designs assuming no feedback 107 errors. In [8] and [9], the performances of equal gain trans108 mission and precoded broadcast transmission were studied, 109 respectively, under the scenario of error-prone limited feedback. 110 Finally, in [10], Housfater and Lim derived a Cramér-Rao-type 111 lower bound for linear precoders. These contributions provided 112 insights into the mitigation of the detrimental impact of the 113 imperfect CSI feedback channel.

114 Another common issue that arises when limited feedback is 115 applied to a typical CoMP system is the codebook design prob116 lem. Although the design of codebooks conceived for limited117 feedback-aided systems has been extensively studied [1], [11], 118 the number of BSs in a CoMP cluster may vary over time, hence 119 requiring a specific design. Thus, it is a challenge to design a 120 codebook imposing low overhead when the number of coop121 erating BSs is high. A promising solution is based on the per122 cell codebook design philosophy of [12]-[15], which separately 123 quantizes the channel associated with each cell within a CoMP 124 channel matrix to avoid a large codebook and to circumvent 125 frequent updates of the codebook owing to either user mobility 126 or due to the different clustering of the BSs. To elaborate a little 127 further, Cheng et al. [12] presented a limited-feedback-based 128 per-cell codebook design and showed that its performance is 129 close to that of the conventional joint-cell codebook design 130 having high overhead. In [13], attention is focussed on the 131 problem of optimal per-cell codebook designs and derived a 132 closed-form solution for the codebook size that minimizes the 133 quantization error on average. In [14], a method of reconstruct134 ing the CoMP channel's CDI was first proposed and then, the 135 performance of different codebook generation techniques and 136 per-cell codeword selection methods was compared.

137 In contrast with the insightful contributions listed earlier, we 138 pursue a different approach in reducing both the algorithmic complexity and the CSI feedback overhead for a scenario where 139 $\mathrm{CS} / \mathrm{CB}$ is employed.

1) We conceive a low-complexity noniterative cross-layer 141 algorithm, which is based on the fact that, in multicell 142 systems, all BSs tend to have different DL-transmission 143 rate requirements and traffic loads. We commence by 144 classifying the BSs into two types. The BSs having higher 145 transmission rate requirements are referred to as master 146 BSs (MBSs), which benefit from a higher priority. The re- 147 maining BSs having lower transmission rate requirements 148 are referred to as slave BSs (SBSs), which have a lower 149 priority.

150

2) We propose a low-complexity interference-threshold- 151 based algorithm for scheduling, which is combined with 152 appropriately adjusting the BF vectors of the cooperating 153 BSs. As shown in Section III, this part of the algorithm 154 only relies on the CSI at the user's side; thus, it is 155 capable of effectively reducing the CSI feedback load 156 while mitigating the inaccuracy of CSI feedback imposed 157 by the error-prone feedback channel.

158

3) Furthermore, we propose a new robust BF vector design 159 for the scenario, where the CDI and CQI are fed back sep- 160 arately. More explicitly, the CDI is quantized before being 161 fed back, whereas the CQI is assumed to be perfectly fed 162 back to the BS.

163

4) We will demonstrate both with the aid of our theoretical 164 derivation and by numerical simulations that our algo- 165 rithm has similar algorithmic complexity as the nonco- 166 operative algorithms. It imposes low backhaul traffic and 167 circumvents the dynamic channel-matrix clustering of 168 CoMP.

5) We also show that the performance of the proposed algo- 170 rithm is not overly compromised and that its performance 171 is similar to that of the iterative algorithm proposed in 172 [16], as far as the MBS is concerned. Our algorithmic 173 philosophy is highlighted in a simple scenario, where 174 only two BSs are involved, but it may be readily ex- 175 tended to more general scenarios supporting multiple BSs 176 without a dramatic increase in complexity and feedback 177 requirements.

The remainder of this paper is organized as follows. In 179 Section II, our system model is introduced, along with some 180 of our basic assumptions. In Section III, both the proposed 181 scheduling and our BF algorithm are detailed. In Section IV, 182 we present a comparison of the algorithmic complexity and the 183 signaling overhead of different algorithms. Our performance 184 analysis is provided in Section V, whereas Section VI offers 185 our conclusions. The proof of some of the theorems is provided 186 in the Appendix.

\section{SySTEM MODEL}

Consider the two-cell network of Fig. 1, where each cell has 189 a BS at its center and multiple MSs scattered within the cell. 190 BS1 on the left of Fig.1 is assumed to be the MBS, and BS2 191 on the right is an SBS. Both BS1 and BS2 are equipped with 192 $K$ transmit antennas, whereas each MS has a single receive 193 antenna. We assume that, each time, each BS schedules its DL 194 


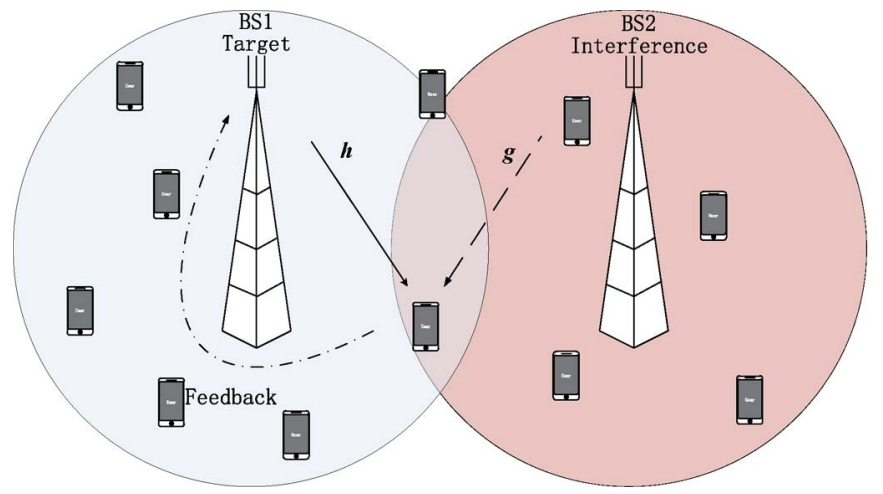

Fig. 1. System model.

195 transmission to a single MS within its cell, and different BSs 196 use the same frequency; hence, the MSs suffer from intercell 197 interference (ICI) imposed by the neighboring BS. The received 198 signal power is related to the location of the MS. There are a 199 number of studies on the fairness control issues of scheduling 200 algorithms [17], [18], but in this paper, we focus our attention 201 on the scenario where the MSs are located at the cell edge, and 202 we assume that all the MSs have the same large-scale fading 203 factor. In other words, the fairness effect of different large-scale 204 fading factors is not considered here.

205 Again, we denote the user set of cell $i(i \in\{1,2\})$ as $\mathcal{U}_{i}$. 206 Then, the signal received by user $j\left(j \in\left\{1,2, \ldots,\left|\mathcal{U}_{1}\right|\right\}\right)$ in cell 2071 and user $k\left(k \in\left\{1,2, \ldots,\left|\mathcal{U}_{2}\right|\right\}\right)$ in cell 2 can be expressed as

$$
\left\{\begin{array}{l}
y_{1, j}=p_{11, j}\left\langle\mathbf{w}_{1}, \mathbf{h}_{1, j}\right\rangle u_{1, j}+p_{21, j}\left\langle\mathbf{w}_{2}, \mathbf{g}_{1, j}\right\rangle u_{2, k}+n_{j} \\
y_{2, k}=p_{22, k}\left\langle\mathbf{w}_{2}, \mathbf{h}_{2, k}\right\rangle u_{2, k}+p_{12, k}\left\langle\mathbf{w}_{1}, \mathbf{g}_{2, k}\right\rangle u_{1, j}+n_{k}
\end{array}\right.
$$

208 where $\langle\mathbf{x}, \mathbf{y}\rangle$ represents the inner product of the vectors $\mathbf{x}$ and $209 \mathbf{y}$. Variable $y_{i, j}$ represents the signal received by user $j$ in 210 cell $i$, where $i$ equals either 1 or 2 . At the receiver of the $j$ th 211 user in cell $i_{1}$, the signal strength received from cell $i_{2}$ can be 212 represented as $p_{i_{1} i_{2}}, j$. The power of the symbol transmitted 213 from BS $i$ to its $j$ th user is denoted $u_{i, j}$, which is normalized 214 as $E\left\{\left|u_{i, j}\right|^{2}\right\}=1$. The random variables $n_{j}$ and $n_{k}$ represent 215 the normalized Gaussian noise, with $E\left\{\left|n_{k}\right|^{2}\right\}=E\left\{\left|n_{j}\right|^{2}\right\}=$ 2161 , whereas vector $\mathbf{h}_{i, j} \in \mathbb{C}^{K \times 1}$ represents the DL channel 217 conditions between $\mathrm{BS} i$ and its $j$ th user, which can be viewed as 218 the target channel condition. Furthermore, vector $\mathbf{g}_{i, j} \in \mathbb{C}^{K \times 1}$ 219 denotes the DL channel condition between the $j$ th user of $220 \mathrm{BS} i$ and the neighboring $\mathrm{BS}$, which is the interferring channel. 221 The target channel vectors and the interfering channel vectors 222 are independent and identically distributed in terms of their 223 statistics, and they both follow a probability distribution of $224 C N\left(0, \mathbf{I}_{K}\right)$. Finally, vector $\mathbf{w}_{i}$ is the BF vector adopted by BSi. 225 The goal of the proposed algorithm is to increase the 226 DL throughput, which is quantified in terms of the channel 227 capacity of

$$
R_{i}=\log \left(1+\operatorname{SINR}_{i}\right)
$$

228 where $\operatorname{SINR}_{i}$ is the signal-to-interference-plus-noise ratio at 229 the scheduled user's terminal of BSi. We assume that each 230 BS schedules its DL transmission to a single MS each time. 231 Thus, to simplify the notation, we denote the target channel condition and the interfering channel condition of the scheduled 232 MS located in cell $i$ by $\mathbf{h}_{i}$ and $\mathbf{g}_{i}$, respectively, yielding

$$
\operatorname{SINR}_{i}=\frac{p_{i i}\left\|\mathbf{h}_{i}\right\|^{2}\left|\left\langle\mathbf{w}_{i}, \tilde{\mathbf{h}}_{i}\right\rangle\right|^{2}}{1+p_{j i}\left\|\mathbf{g}_{i}\right\|^{2}\left|\left\langle\mathbf{w}_{j}, \tilde{\mathbf{g}}_{i}\right\rangle\right|^{2}} .
$$

Note that, in the given equation, the norms of vectors $\mathbf{h}_{i}$ and 234 $\mathrm{g}_{i}$ are separated from their directions so that we have $\left\|\tilde{\mathbf{h}}_{i}\right\|=235$ $\left\|\tilde{\mathbf{g}}_{i}\right\|=1$. We use $p_{i i}$ to denote the signal strength received by 236 the selected user in cell $i$ and $p_{j i}$ to denote the strength of the 237 interfering signal arriving from cell $j$ contaminating the desired 238 user's signal in cell $i$. We simplified the subscript since we only 239 consider the case where each BS schedules its DL transmission 240 to a single user at a time.

\section{A. Schemes Operating Without CoMP}

242

Transmission schemes operating without CoMP typically 243 have lower complexity than those relying on CoMP. Here, 244 we simply consider the classic time-division multiple-access 245 (TDMA) and frequency-division multiple-access (FDMA) 246 schemes. In the TDMA scheme, the $M$ BSs transmit sequen- 247 tially so that each BS transmits in $1 / M$ fraction of the time, 248 without imposing any ICI. In the FDMA scheme, on the other 249 hand, the $M$ BSs share the transmission bandwidth so that each 250 BS transmits in a separate subband without being interfered by 251 the neighboring BSs.

We compare the performance of the TDMA and FDMA 253 schemes to that of our proposed algorithm and to the exhaustive 254 search algorithm. In TDMA and FDMA schemes, all the MSs 255 feed back both their CQI and their target channel conditions 256 using limited feedback, so that the BSs can design BF vectors 257 accordingly. The BSs, on the other hand, schedule their DL 258 transmission for the specific MS having the highest CQI. The 259 transmission throughput for BS $i$ can be expressed as

$$
R_{i}=\frac{1}{M} \log \left(1+\max _{j \in \mathcal{U}_{i}} \operatorname{SNR}_{j}\right)
$$

where $M=2$ in our scenario.

\section{B. Iterative Scheduling and Beamforming}

Iterative algorithms are capable of reducing the algorith- 263 mic complexity while maintaining a similar performance to 264 their exhaustive-search-algorithm-based counterparts. In this 265 paper, we adopt the iterative approach proposed in [16] as a 266 benchmark for our performance evaluation, which relies on the 267 following three steps.

1) Fix the power allocation and scheduled users; then, find 269 the best combination of BF vectors.

2) Fix the BF vectors and power allocation; then, find the 271 best set of users for scheduling.

3) Fix the BF vectors and the scheduled users; then, update 273 the power allocation among the scheduled users.

Since the scenario that we study assumes fixed power allo- 275 cation, the given three-step algorithm reduces to two steps in 276 


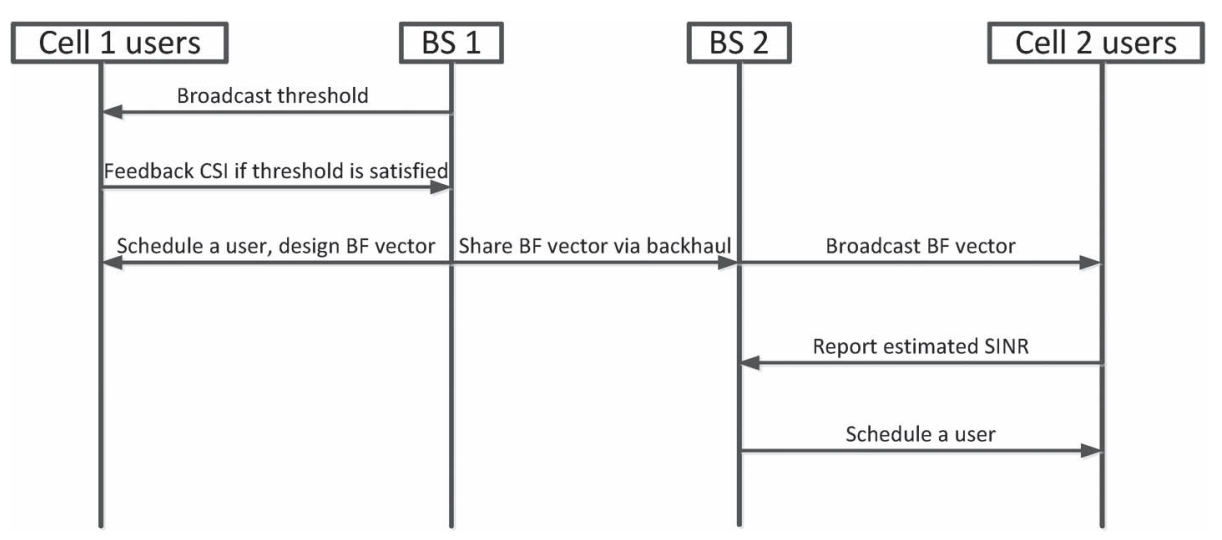

Fig. 2. Algorithm outline.

277 which the combination of BF vectors and the set of scheduled 278 users is updated in an iterative fashion as follows.

1) Fix the set of scheduled users; then, find the optimal set of BF vectors correspondingly.

2) Fix the set of BF vectors; then, find the set of users that yield optimal throughput performance correspondingly.

283 The performance of the algorithm is characterized in 284 Section V. LIMITED FEEDBACK CONSTRAINT

287 The algorithmic steps are shown in Fig. 2. In the first step, 288 the MBS, i.e., BS1, broadcasts a threshold value constraining 289 the channel directions of the desired users with respect to the 290 interfering BS to maintain the target integrity. Explicitly, the 291 directions of the desired user's target channel and of the same 292 user's interfering channel should be perpendicular to each other, 293 which corresponds to the absolute value of their inner product 294 being close to zero. The users within cell 1 receive the threshold 295 value and decide whether or not to feed their CSI back to their 296 anchor BS. If a desired user's target channel and interfering 297 channel satisfy the required angular separation threshold con298 straint, he/she feeds back the CDI of both the target channel and 299 the interfering channel, as well as the CQI of both channels. 300 Here, the CQI is defined as the product of $p_{i i}\left\|\mathbf{h}_{i}\right\|^{2}$ for the 301 target channel and $p_{j i}\left\|\mathbf{g}_{i}\right\|^{2}$ for the interfering channel, when 302 the user is located in cell $i$. Once the CSI of all the users that 303 satisfy the angular separation constraint has been fed back to the $304 \mathrm{MBS}$, the MBS decides which particular user to schedule for its $305 \mathrm{DL}$ transmission and then designs the BF vector, following our 306 robust $\mathrm{BF}$ vector design method to be presented later. Once the $307 \mathrm{BF}$ vector is determined, it is shared with the SBS, i.e., BS2, 308 via a backhaul link, the SBS broadcasts the BF vector, and all 309 the users within cell 2 feed back their expected SINR values 310 computed with the aid of the BF vector and their local CSI. In 311 the final step, the SBS schedules its DL transmissions to the 312 user having the highest "reported" SINR value.

313 The algorithm introduced relies on a few important assump314 tions, which are based on the following motivation.

315 1) Introduction of the constraint $\mathbf{w}_{1}=\mathrm{w}_{2}$. In the original 316 $\mathrm{CS} / \mathrm{CB}$ problem, the $\mathrm{BF}$ vectors of different BSs do not necessarily have the same direction. In fact, if not shared 317 via the backhaul link, the BF vector of a BS can be 318 regarded as a random vector both for the other BSs and for 319 all the MSs of the neighboring cells. There is no simple 320 yet elegant way of effectively reducing the size of the 321 candidate user set, but introducing the given constraint 322 brings us obvious benefits. First, when we have $\mathbf{w}_{1}=323$ $\mathbf{w}_{2}$, the local CSI can be directly used to compute the 324 level of interference, which is now $\left\langle\tilde{\mathbf{h}}_{1}, \tilde{\mathbf{g}}_{2}\right\rangle$ for cell 1 and 325 $\left\langle\tilde{\mathbf{h}}_{2}, \tilde{\mathbf{g}}_{1}\right\rangle$ for cell 2 . This can assists us in exploiting the 326 CSI at the MSs for naturally ruling out DL transmission 327 to the MSs suffering from severe interference. As will be 328 shown in Section IV, the feedback load is substantially 329 reduced. Second, both the scheduling and BF parts of 330 the algorithm can be implemented in each cell by relying 331 merely on local CSI, which means that the backhaul traf- 332 fic is effectively reduced. Additionally, the algorithmic 333 complexity is also significantly cut down since only a 334 small portion of the MBS's users perform CSI feedback, 335 whereas the users of the SBS only feed their CQI back to 336 the BS. These complexity and feedback requirements are 337 similar to or even lower than those of some standard non- 338 CoMP solutions, such as those of the TDMA and FDMA 339 schemes.

2) Introduction of the MBS and the SBS. The related assump- 341 tions are based on the fact that, at each moment, it is 342 likely that some BSs have a higher transmission rate re- 343 quirement than the others; hence, they should be granted 344 a higher priority and, ultimately, a higher transmission 345 rate. Hence, an MBS can schedule its DL transmission 346 to a user and design the BF vector with a higher priority, 347 whereas the SBS cannot. The performance loss imposed 348 by this unbalanced priority can be partially recovered 349 when the number of users in the cells is high.

\section{A. Threshold-Based User Scheduling}

As shown in Fig. 2, the BSs schedule their DL transmissions 352 according to a thresholding algorithm based on a carefully 353 designed threshold. In our proposed algorithm, the MBS and 354 the SBS have the same BF directions. Here, we focus on 355 scheduling the DL transmissions to the specific MS, whose tar- 356 get channel direction $\tilde{\mathbf{h}}$ is "most different" from its interfering 357 


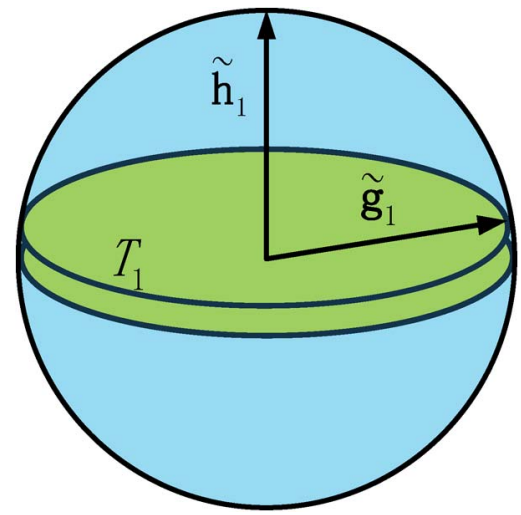

Fig. 3. Threshold.

358 channel direction $\tilde{\mathrm{g}}$ among all the users. More explicitly, we 359 have to find the specific user, whose target channel direction and 360 interfering channel direction have the smallest inner product 361 absolute value across the entire set of users served by the MBS. 362 However, it should be noted that the user to be scheduled for DL 363 transmission in CoMP relying on an exhaustive search might 364 not have an $\tilde{\mathbf{h}}$ perpendicular to $\tilde{\mathrm{g}}$. The thresholds can be defined 365 as follows.

366 The MBS schedules the user whose channel conditions 367 satisfy

$$
\left|\left\langle\tilde{\mathbf{h}}_{1}, \tilde{\mathbf{g}}_{1}\right\rangle\right| \leq T_{1}
$$

368 where $T_{1}$ is the relevant threshold. An intuitive interpretation 369 of the threshold is shown in Fig. 3, which shows (5) with the 370 aid of $T_{1}$. Since the norms of both the BF vectors and of the 371 channel directions of the MSs are 1, these vectors can be placed 372 on a globe-like unit-radius hypersphere in $\mathbb{C}^{K}$, with one end at 373 the origin and the other on the surface of the hypersphere. By 374 assuming that the target channel direction $\tilde{\mathbf{h}}_{1}$ of the scheduled 375 user points to the "north pole" of the globe, the interfering 376 channel direction $\tilde{\mathrm{g}}_{1}$ will fall within the area bounded by the 377 "Tropic," which is characterized by the value of $T_{1}$.

378 Intuitively, when the threshold $T_{1}$ becomes looser, i.e., when 379 it approaches 1, more users will satisfy (5), and the complexity 380 of the algorithm is increased. In particular, when we have $T_{1}=$ 381 1, all the users feed back their CSI, and the algorithm becomes 382 identical to the exhaustive search for the MBS. By contrast, 383 when $T_{1}$ approaches 0 , the scheduling part of the algorithm will 384 guarantee a higher transmission rate for the scheduled users, 385 but it also comes more likely that no users satisfy (5), which 386 leads to lower algorithmic complexity and reduced feedback 387 load. Thus, the threshold controls the tradeoff between the 388 algorithmic complexity and the desired performance; hence, 389 it should be determined under the constraint of ensuring a 390 minimum probability of at least one successful DL scheduling 391 for the entire set of users. The selection of the threshold based 392 upon the given principle can be achieved with the aid of the 393 following theorem.

394 Theorem 1: Let us assume that there are $N_{1}$ users in cell 3951 . Then, for the MBS, the probability of a successful DL 396 scheduling action can be expressed as

$$
P_{\text {suc1 }}=1-\left(1-T_{1}^{2}\right)^{N_{1}(K-1)} .
$$

Proof: See Appendix A.

The performance achieved with the aid of this threshold is 398 characterized in Section IV.

B. Robust Beamforming Under Limited Feedback for the Channel Direction Information

According to Fig. 2, upon scheduling the DL transmission to 402 a user whose channel conditions satisfy (5), the MBS adopts a 403 $\mathrm{BF}$ vector that further improves the throughput. Since the MSs 404 perform limited feedback of their channel conditions, when 405 quantizing both $\tilde{\mathbf{h}}_{1}$ and $\tilde{\mathbf{g}}_{1}$ using a preset codebook and when 406 transmitting the index of a codeword, the quantization error im- 407 poses inaccuracy on the design of BF vectors. We mitigate this 408 impact using a robust BF technique, which maximizes the low- 409 est possible SINR of the specific user selected. Numerous stud- 410 ies have been dedicated to robust BF [19]-[22]. Although the 411 scenarios of these contributions are different, they all model the 412 quantization error as an additive noise vector. For example, 413 the target channel's channel vector of the selected user in cell 1414 would be modeled as

$$
\tilde{\mathbf{h}}_{1}=\hat{\mathbf{h}}_{1}+\mathbf{e}
$$

where $\tilde{\mathbf{h}}_{1}$ represents the actual target channel direction, whereas 416 $\hat{\mathbf{h}}_{1}$ is its quantized version, which is acquired from the user's 417 feedback. Vector e in (7) represents the quantization error, 418 which satisfies the ellipsoid constraint $\|\mathbf{e}\| \leq \varepsilon$. The quanti- 419 zation error for interfering channels can be defined similarly. 420 The problem is then solved using convex optimization, and 421 this technique is assumed to be known in this paper. This 422 traditional way of designing robust BF vectors does not meet 423 the assumptions stipulated in this paper. Earlier, we assumed 424 that the norms of the channel directions are 1 both before and 425 after quantization. This imposes more complex constraints on 426 the description of the quantization error. Hence, we conceive a 427 new technique of designing robust $\mathrm{BF}$ vectors for the scenario 428 when the CDI and CQI are fed back separately.

We adopt the random-vector-quantization codebook concept 430 [23] and use the model of [24] to analyze the quantization error, 431 where the quantization codebook index of B bits is linked with 432 the quantization error by

$$
\left|\left\langle\hat{\mathbf{h}}_{1}, \tilde{\mathbf{h}}_{1}\right\rangle\right| \geq 1-2^{\frac{-B}{K-1}} .
$$

When $\hat{\mathbf{h}}_{1}$ and $\hat{\mathbf{g}}_{1}$ are given, the problem of designing the 434 robust $\mathrm{BF}$ vector can be broken down into two separate parts. $\quad 435$

1) For each $B F$ vector $\mathbf{w}$, find the set of $\tilde{\mathbf{h}}_{1}$ and $\tilde{\mathbf{g}}_{1}$ minimiz- 436 ing the SINR (denoted by $\operatorname{SINR}_{1}$ ) for this $\mathbf{w}$.

2) Find the $B F$ vector $w$, which ensures that this minimized 438 value of $\mathrm{SINR}_{1}$ is maximized.

The whole idea can be formulated as

$$
\mathbf{w}_{\text {opt }}=\arg \max _{\mathbf{w}}\left\{\min _{\tilde{\mathbf{h}}_{1}, \tilde{\mathbf{g}}_{1}}\left\{\operatorname{SINR}_{1}\left(\tilde{\mathbf{h}}_{1}, \tilde{\mathbf{g}}_{1} \mid \hat{\mathbf{h}}_{1}, \hat{\mathbf{g}}_{1}, \mathbf{w}\right)\right\}\right\} .
$$

Again, an intuitive illustration is given in Fig. 4. Since $\tilde{\mathbf{h}}_{1} 441$ and $\tilde{\mathbf{g}}_{1}$ are independent of each other, the problem of finding the 442 specific $\tilde{\mathbf{h}}_{1}$ and $\tilde{\mathbf{g}}_{1}$ that minimize the value of SINR for a given 443 


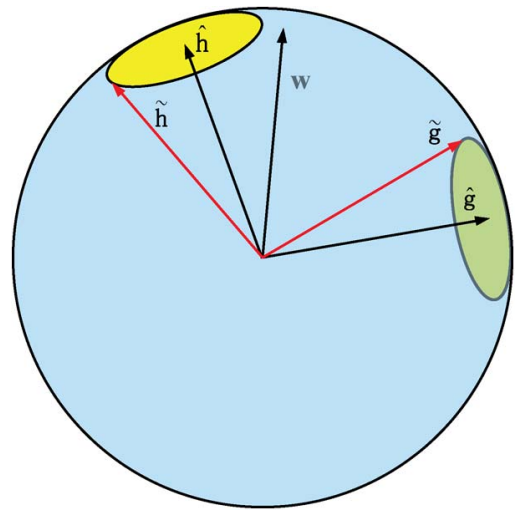

Fig. 4. Robust BF.

$444 \mathbf{w}$ is further simplified to finding $\tilde{\mathbf{h}}_{1}$ that minimizes $\left|\left\langle\mathbf{w}, \tilde{\mathbf{h}}_{1}\right\rangle\right|^{2}$ 445 and finding $\tilde{\mathbf{g}}_{1}$ that maximizes $\left|\left\langle\mathbf{w}, \tilde{\mathbf{g}}_{1}\right\rangle\right|^{2}$. The following lem446 mas provide a way of finding these $\tilde{\mathbf{h}}_{1}$ and $\tilde{\mathbf{g}}_{1}$ vectors.

447 Lemma 1: Upon assuming that $\left|\left\langle\mathbf{w}, \hat{\mathbf{g}}_{1}\right\rangle\right|=\lambda_{1}$ and that the 448 quantization error satisfies $\left|\left\langle\tilde{\mathbf{g}}_{1}, \hat{\mathbf{g}}_{1}\right\rangle\right|=\lambda_{2} \geq \lambda_{2 \min } \geq \lambda_{1}$, we 449 have

$$
\left|\left\langle\mathbf{w}, \tilde{\mathbf{g}}_{1}\right\rangle\right| \leq \lambda_{1} \lambda_{2 \min }+\sqrt{\left(1-\lambda_{1}^{2}\right)\left(1-\lambda_{2 \min }^{2}\right)} .
$$

450 Proof: See Appendix B.

451 Lemma 2: Assuming that $\left|\left\langle\mathbf{w}, \hat{\mathbf{h}}_{1}\right\rangle\right|=\lambda_{1}$ and that the quan452 tization error satisfies $\left|\left\langle\tilde{\mathbf{h}}_{1}, \hat{\mathbf{h}}_{1}\right\rangle\right|=\lambda_{2} \geq \lambda_{2 \min } \geq \sqrt{1-\lambda_{1}^{2}}$, 453 we have

$$
\left|\left\langle\mathbf{w}, \tilde{\mathbf{h}}_{1}\right\rangle\right| \geq \lambda_{1} \lambda_{2 \min }-\sqrt{\left(1-\lambda_{1}^{2}\right)\left(1-\lambda_{2 \min }^{2}\right)} .
$$

454 Proof: See Appendix C.

455 In the given lemmas, $\lambda_{2}$ min $=1-2^{(-B / K-1)}$ represents the 456 maximum quantization error. With the aid of the given lemmas, 457 we have the following theorem.

458 Theorem 2: Upon introducing the notation of

$$
\mathbf{h}_{1}^{\dagger}=\frac{\hat{\mathbf{h}}_{1}-\left\langle\hat{\mathbf{h}}_{1}, \hat{\mathbf{g}}_{1}\right\rangle \hat{\mathbf{g}}_{1}}{\left\|\hat{\mathbf{h}}_{1}-\left\langle\hat{\mathbf{h}}_{1}, \hat{\mathbf{g}}_{1}\right\rangle \hat{\mathbf{g}}_{1}\right\|}
$$

459 and $\hat{\mathbf{h}}_{1}=\delta_{1} \mathbf{h}_{1}^{\dagger}+\delta_{2} \hat{\mathbf{g}}_{1}$, the optimal BF vector can be expressed 460 as a linear combination of vectors $\hat{\mathbf{h}}_{1}$ and $\hat{\mathbf{g}}_{1}$, which is formu461 lated as

$$
\mathbf{w}_{\mathrm{opt}}=\xi_{1} \mathbf{h}_{1}^{\dagger}+\xi_{2} \hat{\mathbf{g}}_{1}
$$

462 where $\xi_{1}$ and $\xi_{2}$ are appropriately chosen so that their norms 463 maximize the SINR in (14), shown at the bottom of the page, 464 whereas the arguments of $\xi_{1}$ and $\xi_{2}$ satisfy the constraint $465 \arg \left(\delta_{1}^{*} \xi_{1}\right)=\arg \left(\delta_{2}^{*} \xi_{2}\right)$.
Proof: See Appendix D. 466

The given theorem provides a numerical technique of design- 467 ing the optimal robust BF vector by finding the optimal value 468 of $\left|\xi_{2}\right|$, which falls in the real-valued interval of $[0,1]$.

\section{Extending the Algorithm to Multiple-BS Scenarios}

Earlier, we developed a low-complexity cross-layer algo- 471 rithm based on a scenario considering only two BSs. Let us 472 now show that this algorithm can be readily extended to more 473 general scenarios with multiple BSs involved.

Let us assume that there are $M$ BSs. In this multi-BS 475 extension of the algorithm, we still assume that there is a 476 single MBS, which cooperates with multiple SBSs, and that 477 all the BSs use the same BF vector. Additionally, since it 478 is reasonable to assume that different BSs have to transmit 479 independent messages to their scheduled users, we can treat the 480 interference at the $j$ th user of cell $k$ received from multiple BSs 481 as interference arriving from a single source associated with a 482 channel vector of

$$
\sum_{i=1, i \neq k}^{M} \mathbf{g}_{i, k, j} u_{i}
$$

where $\mathbf{g}_{i, k, j}$ is the channel vector of the link spanning from the 484 $i$ th BS to the $j$ th user in cell $k$, and $u_{i}$ is the symbol transmitted 485 to the scheduled user in cell $i$. Since the linear combination of 486 isotropic random vectors is also isotropic, the threshold-based 487 scheduling of Section III-A remains unchanged.

\section{Algorithmic Complexity AND SIGNALING OVERHEAD}

Here, we characterize the complexity of four algorithms: the 491 exhaustive search algorithm, the TDMA/FDMA scheme, the 492 iterative algorithm of [16], and our proposed algorithm.

493

Consider the scenario associated with $M$ BSs. The search for 494 the BF vector of a single BS is based on a searching through an 495 $L$-entry codebook, as implied in (14). Therefore, the exhaustive 496 search algorithm has complexity of $\mathcal{O}\left(L^{M} \prod_{i=1}^{M}\left|\mathcal{U}_{i}\right|\right)$, where 497 $\left|\mathcal{U}_{i}\right|$ is the supported number of users in the $i$ th BS, because 498 the algorithm will consider all possible user combinations to 499 find the one that achieves the optimal performance, which is 500 estimated based on an exhaustive search through the codebook 501 of each BS for an optimal combination of BF vectors for each 502 possible user combination.

503

The complexity of the iterative algorithm proposed in [16] 504 cannot be readily determined without knowing the number 505 of iterations needed for the convergence of the scheduling 506 process. Here, we introduce parameter $S$, which represents 507

$$
\operatorname{SINR}_{1}=\frac{\left.p_{11}\left\|\mathbf{h}_{1}\right\|^{2}\left(\lambda_{2 \min }\left(\left|\delta_{1} \xi_{1}\right|+\left|\delta_{2} \xi_{2}\right|\right)-\sqrt{\left(1-\lambda_{2 \min }^{2}\right)\left(1-\left(\left|\delta_{1} \xi_{1}\right|+\left|\delta_{2} \xi_{2}\right|\right)^{2}\right.}\right)\right)^{2}}{1+p_{21}\left\|\mathbf{g}_{1}\right\|^{2}\left(\left|\lambda_{2 \min } \xi_{2}\right|+\sqrt{\left(1-\lambda_{2 \min }^{2}\right)\left(1-\left|\xi_{2}\right|^{2}\right)}\right)^{2}}
$$


TABLE I

ALGORITHMIC COMPLEXITY

\begin{tabular}{|c|c|c|c|c|}
\hline Algorithm & Exhaustive Search & Iterative & TDMA/FDMA & Proposed \\
\hline Time(s) & $1.34 \mathrm{e} 3$ & $1.50 \mathrm{e} 1$ & $2.15 \mathrm{e}-1$ & $6.04 \mathrm{e}-1$ \\
\hline Complexity & $\mathcal{O}\left(L^{M} \prod_{i=1}^{M}\left|\mathcal{U}_{i}\right|\right)$ & $\mathcal{O}\left(S\left(L^{M}+\prod_{i=1}^{M}\left|\mathcal{U}_{i}\right|\right)\right)$ & $\mathcal{O}\left(\sum_{i=1}^{M}\left|\mathcal{U}_{i}\right|\right)$ & $\mathcal{O}\left(\left|\mathcal{U}_{1}\right| L+\sum_{i=2}^{M}\left|\mathcal{U}_{i}\right|\right)$ \\
\hline
\end{tabular}

TABLE II

SIGNALING OVERHEAD

\begin{tabular}{|c|c|c|c|c|c|c|}
\hline \multirow{2}{*}{\multicolumn{2}{|c|}{ Algorithm }} & \multirow{2}{*}{ Exhaustive search } & \multirow{2}{*}{ Iterative } & \multirow{2}{*}{ TDMA/FDMA } & \multicolumn{2}{|c|}{ Proposed } \\
\hline & & & & & BS1 (MBS) & $\mathrm{BS} i$ (SBS) \\
\hline \multicolumn{2}{|c|}{ CDI feedback per user } & $2 M$ & $2 M$ & 0 & 2 & 0 \\
\hline \multicolumn{2}{|c|}{ CQI feedback per user } & $2 M$ & $2 M$ & 1 & 2 & 1 \\
\hline \multicolumn{2}{|c|}{ Number of feedback users } & $\left|\mathcal{U}_{i}\right|$ & $\left|\mathcal{U}_{i}\right|$ & $\left|\mathcal{U}_{i}\right|$ & Greatly reduced & $\left|\mathcal{U}_{i}\right|$ \\
\hline \multirow{4}{*}{ Backhaul traffic } & CDI exchange & $\sum^{M} M\left|\mathcal{U}_{i}\right|$ & $M^{2}$ & 0 & \multicolumn{2}{|l|}{ 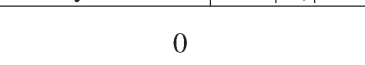 } \\
\hline & CQI exchange & $\sum_{i=1}^{M} M\left|\mathcal{U}_{i}\right|$ & $M^{2}$ & 0 & \multicolumn{2}{|c|}{0} \\
\hline & BF vector exchange & $M$ & $M$ & 0 & \multicolumn{2}{|c|}{$M-1$} \\
\hline & User identity exchange & $M$ & $M$ & 0 & \multicolumn{2}{|c|}{0} \\
\hline
\end{tabular}

508 the maximum affordable number of iterations for the algo509 rithm. Then, the resultant complexity may be expressed as $510 \mathcal{O}\left(S\left(L^{M}+\prod_{i=1}^{M}\left|\mathcal{U}_{i}\right|\right)\right)$, which follows from the fact that, each 511 time the BF vectors are updated, an exhaustive search of the 512 codebooks is conducted, whereas an exhaustive search for 513 the optimal user combination is carried out every time, when 514 the combination is updated.

515 The complexity of the TDMA/FDMA algorithms is $516 \mathcal{O}\left(\sum_{i=1}^{M}\left|\mathcal{U}_{i}\right|\right)$, which follows from the fact that each BS oper517 ates on its own and only has to set the BF vector to the direction 518 of the target channel of the scheduled user.

519 For the proposed algorithm, if we assume that BS1 is 520 the MBS, the upper bound of the complexity is $\mathcal{O}\left(\left|\mathcal{U}_{1}\right| L+\right.$ $\left.521 \sum_{i=2}^{M}\left|\mathcal{U}_{i}\right|\right)$. This is because the MBS only has a portion of 522 its users performing feedback, and the SBSs only have to find 523 the best users on their own by comparing their CQI feedback 524 values.

525 In the following, we characterize the algorithmic complexity 526 by monitoring the simulation time required for a single trial. We 527 conducted 1000 trials on all the algorithms aforementioned, and 528 in Table I, we listed the time required for each single trial for 529 CDI codebook sizes spanning from 2 to 15 . As we can see, the 530 exhaustive search algorithm and the iterative algorithm require 531 significantly more time than the noncooperative algorithm and 532 the proposed algorithm.

533 Finally, we compare the signaling overhead of the four given 534 algorithms in Table II, which includes the overhead generated 535 from users' feedback and the backhaul traffic. It is observed in 536 Table II that the exhaustive search algorithm and the iterative 537 algorithm require the same amount of feedback overhead, ex538 cept that the iterative algorithm generates less backhaul traffic 539 when the number of iterations is small. The overhead generated, 540 particularly the backhaul traffic, is enormous compared with the 541 noncooperative TDMA/FDMA schemes.

542 The proposed algorithm, on the other hand, effectively con543 trols the overhead generated from the CSI feedback and back544 haul traffic. Since the BF vectors are set to the same direction, 545 each user can view its received signals as if they were sent from only two sources, i.e., the destination and a single interfering 546 source. As a benefit, each user only has to feed back two sets of 547 channel information. Furthermore, the number of users generat- 548 ing feedback for the MBS is substantially reduced. A theoretical 549 expression for this number is hard to derive. Nonetheless, we 550 can determine with the aid of numerical simulations that, when 551 there are 20 users in each cell and the successful transmission 552 ratio is set to $99.9 \%$, the number of feedback users in the MBS 553 is, on average, 5.7. Meanwhile, the proposed algorithm only 554 requires the sharing of the BF vector selected by the MBS, 555 which is almost negligible compared with the backhaul traffic 556 generated by the iterative algorithm.

\section{Numerical Results}

Here, we first characterize the properties of threshold $T_{1}$, and 559 then quantify the performance of our proposed algorithm. $\quad 560$

\section{A. Threshold Value}

According to (5) and (6), the "Tropic" $T_{1}$ controls the trade- 562 off between the feedback load, algorithmic complexity, and the 563 probability of a successful DL scheduling action. From (6), $T_{1} 564$ is also a function of the number of transmit antennas and the 565 number of active users within the cell.

Fig. 5 shows the relation between the number of users and 567 the value of $T_{1}$, where the number of transmit antennas was set 568 to 2. Parameters $p_{i i}$ and $p_{j i}$ of both the MBS and the SBS are 569 set to $30 \mathrm{~dB}$, whereas the probability of success ranged from 570 $10 \%$ to $99.9 \%$. We can see in Fig. 5 that, when the probability 571 of success decreases, the threshold becomes stricter, and when 572 the number of users increases, the value of $T_{1}$ approaches 0.573 This is a manifestation of multiuser diversity since we are more 574 likely to have a user with better channel conditions when the 575 number of users becomes larger.

Fig. 6 shows the relationship between the probability of 577 successful DL scheduling and the percentage of reduction in 578 the CDI feedback per user. The numbers of users in both cells 579 range from 10 to 40 , whereas the number of transmit antennas 580 


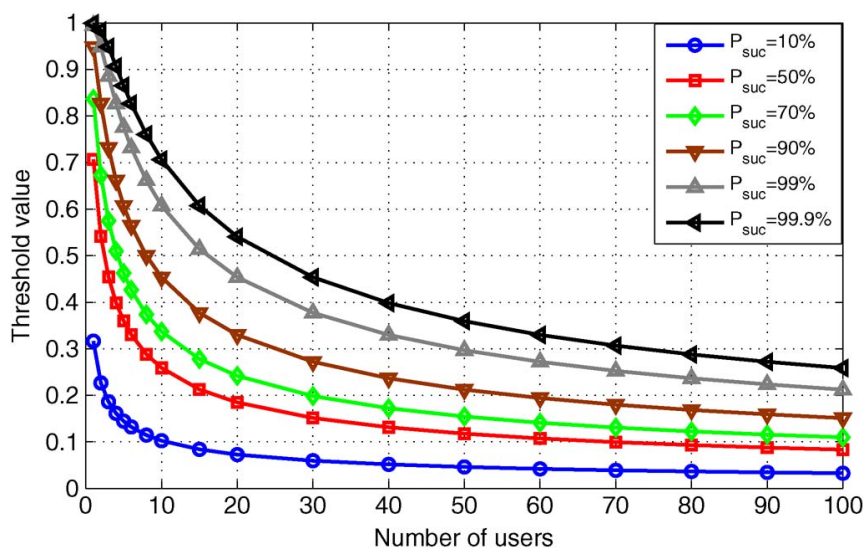

Fig. 5. Threshold value.

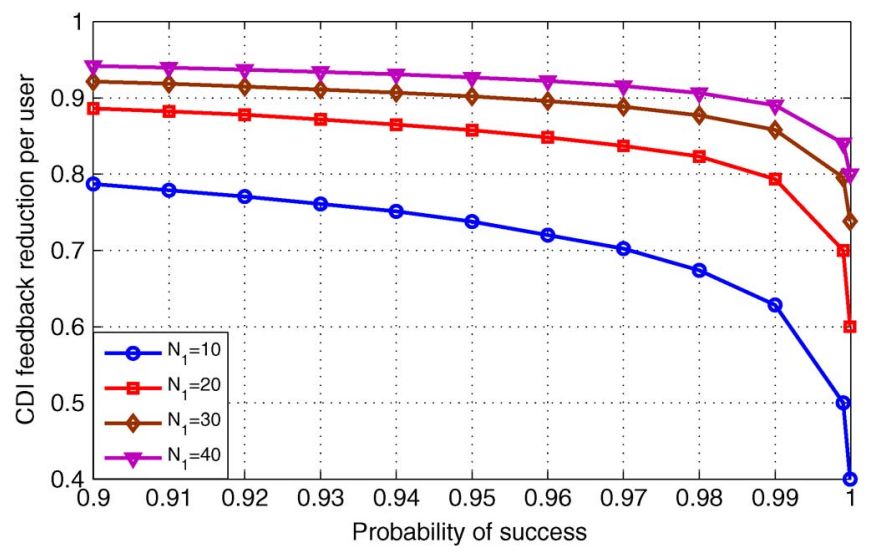

Fig. 6. Feedback load reduction per user.

581 remains 2, and the values $p_{i i}$ and $p_{j i}$ of both the MBS and the 582 SBS remain $30 \mathrm{~dB}$. Note that, in traditional TDMA and FDMA 583 schemes, all the users feed back their CSI; hence, the feedback 584 load per user is one CDI, where the number of bits is determined 585 by the codebook size. In CS/CB relying on exhaustive search, 586 the feedback load per user is two CDI times the number of 587 codebook index bits. In our proposed algorithm, however, the 588 feedback load per user is only $0.3 \mathrm{CDI}$, when the number of 589 users is 20 in both cells and the probability of success is $99.9 \%$. 590 This implies that, compared with TDMA and FDMA schemes, 591 the threshold $T_{1}$ allows us to rule out $70 \%$ of feedback without 592 undue degradation of the probability of success. Additionally, it 593 is possible to achieve an even lower feedback load by reducing 594 the probability of a successful DL scheduling, when the number 595 of users is given, but an excessive degradation of the probability 596 of success may ultimately impose throughput degradation.

\section{B. Throughput Performance}

598 Fig. 7 quantifies the throughput of the MBS relying on the 599 proposed algorithm in comparison to both the exhaustive search 600 scheme and the traditional TDMA and FDMA schemes. The 601 iterative algorithm proposed in [16] is used as a benchmark. 602 The number of users in both cells was set to 20, whereas 603 the parameters $p_{i i}$ and $p_{j i}$ of both the target $B S$ and of the 604 interfering $B S$ were set to $30 \mathrm{~dB}$, which was attenuated by 605 the path loss. The number of transmit antennas was two, and

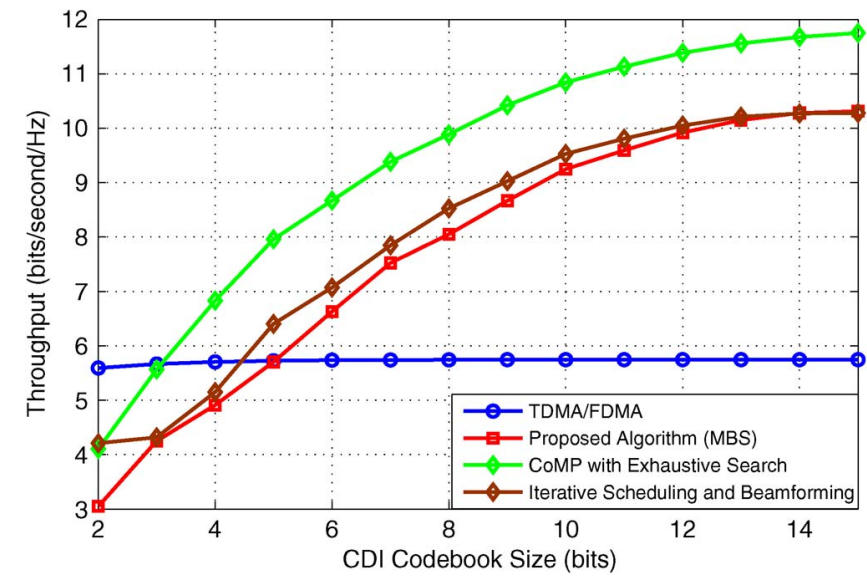

Fig. 7. DL throughput performance of the MBS.

the probability of DL scheduling success was set to $99.9 \% .606$ As shown in Fig. 7, the performance of the MBS does not 607 substantially degrade, and it is similar to the performance of the 608 iterative algorithm proposed in [16]. The discrepancy between 609 the exhaustive search algorithm and our proposed algorithm is a 610 result of striking a tradeoff between the algorithmic complexity, 611 overhead, and performance. Again, the discrepancy between 612 the iterative algorithm and the exhaustive search algorithm is 613 contributed by both the imperfect CSI feedback and by the 614 fact that the iterative algorithm cannot guarantee consistent 615 convergence to the global optimum. We also note that the left- 616 hand part of the curves is not as smooth as their right-hand 617 part. When the size of the codebook is 2 bits, the iterative 618 algorithm even yields a slightly better performance than the 619 exhaustive search algorithm. This, however, does not mean 620 that the iterative algorithm performs in general better than the 621 exhaustive search algorithm. This phenomenon is due to the 622 large quantization errors of the CDI feedback. While the ex- 623 haustive search algorithm did guarantee the maximization of the 624 minimum throughput of the scheduled users, the maximization 625 of the actual performance is evaluated with the aid of the actual 626 channel conditions encountered by the scheduled users. Hence, 627 we conclude that the better performance of the iterative algo- 628 rithm is a result of the large quantization errors imposed by the 629 limited codebook size of the CDI feedback. This phenomenon 630 does not occur when the codebook size is large.

631

Furthermore, observe in Fig. 7 that when the CDI codebook 632 size is small, the performance of cooperative $\mathrm{BF}$ is similar to or 633 even lower than that of the noncooperative TDMA and FDMA 634 schemes. This phenomenon raises the question as to what is the 635 minimum amount of feedback required by cooperative trans- 636 mission and scheduling schemes to outperform their traditional 637 noncooperative counterparts. This question is studied in detail 638 in [15].

Fig. 8 shows the performance comparison of an SBS and 640 the performance of a regular BS running under TDMA/FDMA 641 schemes. The codebook size is set to 15 in this case, whereas 642 the performances with other codebook sizes are similar. It can 643 be easily seen that the performance of the SBS is sometimes 644 not as good as the regular BS but becomes better as the number 645 of users increases. This is caused by the multiuser diversity 646 effect. It can be also observed that the intersection of the 647 


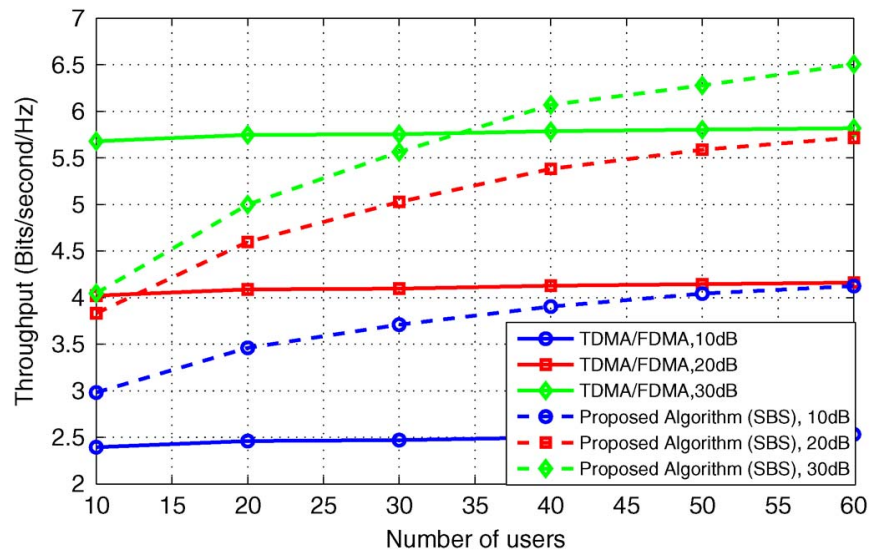

Fig. 8. Performance comparison between an SBS and a BS running under TDMA/FDMA schemes.

648 curves of the performances of the SBS and the regular BS 649 shifts toward right as the SNR increases, which means that at 650 high SNR levels, the proposed scheme will need more users 651 to beat the performance of the TDMA/FDMA schemes. This 652 phenomenon can be interpreted by the suboptimality of the $653 \mathrm{BF}$ vector. For a small amount of the given users, the effect 654 of $\mathrm{BF}$ is dominating when SNR level is low. However, as 655 SNR increases, the suboptimality of the direction of the BF 656 vector becomes a major constraint to improve the performance, 657 and the proposed algorithm is surpassed by the TDMA/FDMA 658 schemes gradually.

\section{CONCLUSION}

660 In this paper, we have conceived a low-complexity cross661 layer algorithm for DL CoMP, which promises a good per662 formance for the MBS while significantly reducing both the 663 amount of feedback and the algorithmic complexity. The 664 scheduling scheme of the proposed algorithm efficiently ex665 ploited the knowledge of CSI at the receiver. For the BF part of 666 the algorithm, we provided a new technique of designing robust $667 \mathrm{BF}$ vectors, when the CDI and CQI of the users are fed back 668 to the BSs separately. Our numerical results demonstrated that 669 our solution only moderately degraded the performance of the 670 potentially excessive-complexity exhaustive search technique, 671 despite having complexity as low as that of a conventional 672 system operating without CoMP. We next present the proofs 673 of the theorems stated earlier.

674

675

676 For the MBS, denote the channel directions of the sched677 uled user as $\tilde{\mathbf{h}}_{1}=\left[h_{1}, h_{2}, \ldots, h_{K}\right]^{T}, \tilde{\mathbf{g}}_{1}=\left[g_{1}, g_{2}, \ldots, g_{K}\right]^{T}$. 678 Since $\mathbf{h}_{1} \sim C N\left(0, \mathbf{I}_{K}\right)$, it follows that $\left[\operatorname{Re}\left(h_{i}\right), \operatorname{Im}\left(h_{i}\right)\right]^{T} \sim$ $679 N\left(0,\left(1 / 2 \mathbf{I}_{2}\right)\right.$ and $\left[\operatorname{Re}\left(g_{i}\right), \operatorname{Im}\left(g_{i}\right)\right]^{T} \sim N\left(0,(1 / 2) \mathbf{I}_{2}\right)$. Thus, 680 the random vector $\tilde{\mathbf{h}}_{1}$ spanning the complex space $\mathbb{C}^{K}$ equals

${ }^{1}$ We discovered that similar results are derived in [25], where the authors directly computed the surface area of the unit hypersphere and spherical cap in complex space. The absolute surface area is not the same when considered in a $K$-D complex space and a $2 K$-D real space, but the resulting probability is the same. a random vector confined to the real space, which can be 681 formulated as $\overline{\mathbf{h}}_{1} \in \mathbb{R}^{2 K}$. The real random vector $\overline{\mathbf{h}}_{1}$ obeys the 682 normal distribution of $N\left(0,(1 / 2) \mathbf{I}_{2 K}\right)$.

According to Section III-A, the goal of the scheduling al- 684 gorithm is to find the specific user, whose channel directions 685 $\widetilde{\mathbf{h}}_{1}$ and $\tilde{\mathbf{g}}_{1}$ are "most different" from each other. Assuming 686 $\left|\left\langle\tilde{\mathbf{h}}_{1}, \tilde{\mathbf{g}}_{1}\right\rangle\right|=|\cos \theta|$, the probability density function of $|\cos \theta| 687$ can be expressed as

$$
f(|\cos \theta|)=f\left(\left|\sum_{i=1}^{K} h_{i} g_{i}^{*}\right|\right)=f\left(\sqrt{A_{3}^{2}+A_{4}^{2}}\right)
$$

where $f(\cdot)$ denotes the probability density function of any 689 random variable or random vector, and

$$
\begin{aligned}
& A_{3}=\sum_{i=1}^{K}\left[\operatorname{Re}\left(h_{i}\right) \operatorname{Re}\left(g_{i}\right)+\operatorname{Im}\left(h_{i}\right) \operatorname{Im}\left(g_{i}\right)\right] \\
& A_{4}=\sum_{i=1}^{K}\left[\operatorname{Re}\left(h_{i}\right) \operatorname{Im}\left(g_{i}\right)-\operatorname{Re}\left(g_{i}\right) \operatorname{Im}\left(h_{i}\right)\right] .
\end{aligned}
$$

Now, if we define three new random vectors in the set of $\mathbb{R}^{2 K} 691$

$$
\begin{aligned}
\overline{\mathbf{h}}^{(1)} & =\left[\operatorname{Re}\left(h_{1}\right), \ldots, \operatorname{Re}\left(h_{K}\right), \operatorname{Im}\left(h_{1}\right), \ldots, \operatorname{Im}\left(h_{K}\right)\right]^{T} \\
\overline{\mathbf{g}}^{(1)} & =\left[\operatorname{Re}\left(g_{1}\right), \ldots, \operatorname{Re}\left(g_{K}\right), \operatorname{Im}\left(g_{1}\right), \ldots, \operatorname{Im}\left(g_{K}\right)\right]^{T} \\
\overline{\mathbf{g}}^{(2)} & =\left[\operatorname{Im}\left(g_{1}\right), \ldots, \operatorname{Im}\left(g_{K}\right),-\operatorname{Re}\left(g_{1}\right), \ldots,-\operatorname{Re}\left(g_{K}\right)\right]
\end{aligned}
$$

then, (16) can be further simplified as

$$
f(|\cos \theta|)=f\left(\sqrt{\left|\left(\overline{\mathbf{h}}^{(1)}\right)^{T} \overline{\mathbf{g}}^{(1)}\right|^{2}+\left|\left(\overline{\mathbf{h}}^{(1)}\right)^{T} \overline{\mathbf{g}}^{(2)}\right|^{2}}\right) .
$$

Since we have $\left|\left(\overline{\mathbf{g}}^{(1)}\right)^{T} \overline{\mathbf{g}}^{(2)}\right|=0$, there exists $(2 K-693$ 2) real-valued vectors with unit norms of $\overline{\mathbf{g}}^{(3)}, \ldots, \overline{\mathbf{g}}^{(2 K)}, 694$ which are orthogonal to each other, including $\overline{\mathbf{g}}^{(1)}$ and 695 $\overline{\mathbf{g}}^{(2)}$. Thus, by letting $\mathbf{M}=\left[\overline{\mathbf{g}}^{(1)}, \overline{\mathbf{g}}^{(2)}, \ldots, \overline{\mathbf{g}}^{(2 K)}\right]$, we have 696 $\left|\left(\overline{\mathbf{h}}^{(1)}\right)^{T} \mathbf{M} \mathbf{M}^{T} \overline{\mathbf{g}}^{(1)}\right|^{2}=\operatorname{Re}\left(h_{1}\right)^{2}$ and $\left|\left(\overline{\mathbf{h}}^{(1)}\right)^{T} \mathbf{M M}^{T} \overline{\mathbf{g}}^{(2)}\right|^{2}=697$ $\operatorname{Im}\left(h_{1}\right)^{2}$; thus

$f(|\cos \theta|)=f\left(\sqrt{\operatorname{Re}\left(h_{1}\right)^{2}+\operatorname{Im}\left(h_{1}\right)^{2}} \mid \overline{\mathbf{g}}^{(1)}\right) f\left(\overline{\mathbf{g}}^{(1)}\right)$.

This means that the value of $|\cos \theta|^{2}$ equals the sum of the 699 squares of the two coordinates of $\overline{\mathbf{h}}^{(1)}$ along the two orthog- 700 onal dimensions. Additionally, note that the direction of the 701 random vector $\overline{\mathbf{h}}^{(1)}$ is isotropic [26], which implies that the 702 probability density function of $\tilde{\mathbf{h}}_{1}$ on the surface of the $2 K$-D 703 hypersphere with unit radius is $1 / S_{2 K}$, where we have 704 $S_{2 K}=2 K \pi^{K} / \Gamma(1+K)$. If we define $S_{\operatorname{Re}\left(h_{1}\right)^{2}+\operatorname{Im}\left(h_{1}\right)^{2} \leq T_{1}^{2} 705}$ and $S_{\operatorname{Re}\left(h_{1}\right)^{2}+\operatorname{Im}\left(h_{1}\right)^{2}=r^{2}}$ to be the surface area of the hyper- 706 sphere satisfying the constraint described in the subscript, the 707 probability that a specific user's channel directions satisfy the 708 threshold constraint denoted by $P_{1}$ can be expressed as

$$
\begin{aligned}
P_{1} & =P\left(\sqrt{\operatorname{Re}\left(\mathrm{h}_{1}\right)^{2}+\operatorname{Im}\left(\mathrm{h}_{1}\right)^{2}} \leq T_{1}\right) \\
& =\frac{S_{\operatorname{Re}\left(h_{1}\right)^{2}+\operatorname{Im}\left(h_{1}\right)^{2} \leq T_{1}^{2}}}{S_{2 K}} .
\end{aligned}
$$


710 By exploiting

$$
\begin{aligned}
& S_{\operatorname{Re}\left(h_{1}\right)^{2}+\operatorname{Im}\left(h_{1}\right)^{2} \leq T_{1}^{2}} \\
& =\int_{0}^{T_{1}} S_{\operatorname{Re}\left(h_{1}\right)^{2}+\operatorname{Im}\left(h_{1}\right)^{2}=r^{2} d r} \\
& =\int_{0}^{\arcsin T_{1}}\left[\frac{2(K-1) \pi^{K-1} r^{2 K-3}}{\Gamma(K)}\right]_{r=\cos \theta} 2 \pi \sin \theta d \theta \\
& =\frac{2 \pi^{K}\left(1-\left(1-T_{1}^{2}\right)^{K-1}\right)}{\Gamma(K)}
\end{aligned}
$$

711 we arrive at

$$
P_{1}=1-\left(1-T_{1}^{2}\right)^{K-1}
$$

712 Since there are $N_{1}$ users in the cell, the probability that there 713 is at least one user that satisfies the threshold constraint can be 714 expressed as

$$
P_{\text {suc1 }}=1-\left(1-P_{1}\right)^{N_{1}} \text {. }
$$

715 With the aid of (23), we finally have

$$
P_{\text {suc } 1}=1-\left(1-T_{1}^{2}\right)^{N_{1}(K-1)} .
$$

\section{6 \\ 717 \\ APPENDIX B \\ PROOF OF LEMMA 1}

718 Let

$$
\mathbf{w}_{1}=\frac{\mathbf{w}-\left\langle\mathbf{w}, \hat{\mathbf{g}}_{1}\right\rangle \hat{\mathbf{g}}_{1}}{\left\|\mathbf{w}-\left\langle\mathbf{w}, \hat{\mathbf{g}}_{1}\right\rangle \hat{\mathbf{g}}_{1}\right\|}
$$

719 Then, $\mathbf{w}_{1}$ is orthogonal to $\hat{\mathbf{g}}_{1}$, and it can be expressed as a linear 720 combination of $\hat{\mathbf{g}}_{1}$ and $\mathbf{w}$. Thus, we can assume that

$$
\begin{aligned}
& \mathbf{w}=\varphi_{1} \mathbf{w}_{1}+\varphi_{2} \hat{\mathbf{g}}_{1} \\
& \tilde{\mathbf{g}}_{1}=\theta_{1} \mathbf{w}_{1}+\theta_{2} \hat{\mathbf{g}}_{1}+\theta_{3} \mathbf{u}
\end{aligned}
$$

721 where vector $\mathbf{u}$ is an arbitrary vector with unit norm and 722 orthogonal to both $\hat{\mathbf{g}}_{1}$ and $\mathbf{w}$. Additionally, we should also note 723 that, in the given equations

$$
\left|\varphi_{1}\right|^{2}+\left|\varphi_{2}\right|^{2}=\left|\theta_{1}\right|^{2}+\left|\theta_{2}\right|^{2}+\left|\theta_{3}\right|^{2}=1 \text {. }
$$

724 Thus, we have

$$
\left|\left\langle\mathbf{w}, \tilde{\mathbf{g}}_{1}\right\rangle\right|=\left|\varphi_{1} \theta_{1}^{*}+\varphi_{2} \theta_{2}^{*}\right| \leq\left|\varphi_{1} \theta_{1}\right|+\left|\varphi_{2} \theta_{2}\right|
$$

725 where the equality on the right side holds if and only if we have

$$
\arg \left(\varphi_{1} \theta_{1}^{*}\right)=\arg \left(\varphi_{2} \theta_{2}^{*}\right)
$$

726 According to (28), we have

$$
\left|\left\langle\tilde{\mathbf{g}}_{1}, \hat{\mathbf{g}}_{1}\right\rangle\right|=\left|\theta_{2}\right|=\lambda_{2} \geq \lambda_{2 \min } \geq \lambda_{1}=\left|\varphi_{2}\right| \text {. }
$$

We can see from the given equation that the maximum 727 value of $\left|\left\langle\mathbf{w}, \tilde{\mathbf{g}}_{1}\right\rangle\right|$ is achieved when $\theta_{3}$ is zero. If not, we 728 can force $\theta_{3}$ to zero and multiply both $\theta_{1}$ and $\theta_{2}$ by a factor 729 of $1 / \sqrt{1-\left|\theta_{3}\right|^{2}}$. According to (30), the value of $\left|\left\langle\mathbf{w}, \tilde{\mathbf{g}}_{1}\right\rangle\right| 730$ increases. Since $\left|\theta_{2}\right|$ is always larger than $\left|\varphi_{2}\right|$, according to 731 Chebyshev's inequality, we can see that increasing the value 732 of $\left|\varphi_{2}\right|$ will result in a monotonic increase in the value of 733 $\left|\left\langle\mathbf{w}, \tilde{\mathbf{g}}_{1}\right\rangle\right|$. Thus, the maximum value of $\left|\left\langle\mathbf{w}, \tilde{\mathbf{g}}_{1}\right\rangle\right|$ is achieved, 734 when $\lambda_{2}=\lambda_{2 \text { min. }}$. This means that the maximum value of 735 $\left|\left\langle\mathbf{w}, \tilde{\mathbf{g}}_{1}\right\rangle\right|$ is achieved, when the actual channel direction falls 736 on the quantization cell boundary between the BF vector and 737 the unit vector representing the quantized channel direction. 738

\section{APPENDIX C \\ PROOF OF LEMMA 2}

Lemma 2 can be proven in the same way as Lemma 1. Let 741

$$
\mathbf{w}_{1}=\frac{\mathbf{w}-\left\langle\mathbf{w}, \hat{\mathbf{h}}_{1}\right\rangle \hat{\mathbf{h}}_{1}}{\left\|\mathbf{w}-\left\langle\mathbf{w}, \hat{\mathbf{h}}_{1}\right\rangle \hat{\mathbf{h}}_{1}\right\|} .
$$

Then, vector $\mathbf{w}_{1}$ is orthogonal to $\hat{\mathbf{h}}_{1}$, and it can be expressed as 742 a linear combination of $\mathbf{w}$ and $\hat{\mathbf{h}}_{1}$. Thus, we can assume that 743

$$
\begin{aligned}
\mathbf{w} & =\varphi_{3} \mathbf{w}_{1}+\varphi_{4} \hat{\mathbf{h}}_{1} \\
\tilde{\mathbf{h}}_{1} & =\theta_{4} \mathbf{w}_{1}+\theta_{5} \hat{\mathbf{h}}_{1}+\theta_{6} \mathbf{u}
\end{aligned}
$$

where vector $\mathbf{u}$ is an arbitrary vector with a unit norm and 744 orthogonal to both $\hat{\mathbf{h}}_{1}$ and $\mathbf{w}$. Since the norms of both $\mathbf{w}$ and 745 $\tilde{\mathbf{h}}_{1}$ are 1 , it easily follows that

$$
\left|\varphi_{3}\right|^{2}+\left|\varphi_{4}\right|^{2}=\left|\theta_{4}\right|^{2}+\left|\theta_{5}\right|^{2}+\left|\theta_{6}\right|^{2}=1 \text {. }
$$

Thus, we have

$$
\left|\left\langle\mathbf{w}, \tilde{\mathbf{h}}_{1}\right\rangle\right|=\left|\varphi_{3} \theta_{4}^{*}+\varphi_{4} \theta_{5}^{*}\right| \geq\left\|\varphi_{3} \theta_{4}|-| \varphi_{4} \theta_{5}\right\| .
$$

It is clear from (34) and (35) that $\left|\varphi_{4}\right|=\left|\lambda_{1}\right|$ and $\left|\theta_{5}\right|=\lambda_{2} .748$ Since $\lambda_{2} \geq \sqrt{1-\lambda_{1}^{2}}$, we have $\lambda_{1} \geq \sqrt{1-\lambda_{2}^{2}}$. Thus

$$
|| \varphi_{3} \theta_{4}|-| \varphi_{4} \theta_{5} \|=\lambda_{1} \lambda_{2}-\sqrt{\left(1-\lambda_{1}^{2}\right)\left(1-\lambda_{2}^{2}-\left|\theta_{6}\right|^{2}\right)} .
$$

In the given equation, we first observe that when $\left|\left\langle\mathbf{w}, \tilde{\mathbf{h}}_{1}\right\rangle\right| 750$ is minimized, $\left|\theta_{6}\right|$ has to be zero. This is obvious since, 751 for a given $\lambda_{1}$, if we hold $\lambda_{2}$ at a fixed value smaller 752 than 1 , increasing the value of $\left|\theta_{6}\right|$ will result in a de-753 crease in $\sqrt{\left(1-\lambda_{1}^{2}\right)\left(1-\lambda_{2}^{2}-\left|\theta_{6}\right|^{2}\right)}$, and if the value of 754 $\left|\left\langle\mathbf{w}, \tilde{\mathbf{h}}_{1}\right\rangle\right|$ is minimized, $\left|\theta_{6}\right|$ increases. Additionally, when 755 $\left|\left\langle\mathbf{w}, \tilde{\mathbf{h}}_{1}\right\rangle\right|$ is minimized, $\left|\theta_{6}\right|$ is minimized, and $\lambda_{2}=\lambda_{2 \min } .756$ This is because when $\lambda_{2}$ decreases, $\lambda_{1} \lambda_{2}$ decreases, and 757 $\sqrt{\left(1-\lambda_{1}^{2}\right)\left(1-\lambda_{2}^{2}-\left|\theta_{6}\right|^{2}\right)}$ increases at the same time.

758

With the given observations, the lemma is proven. Note that 759 this lemma tells us that the minimum of $\left|\left\langle\mathbf{w}, \tilde{\mathbf{h}}_{1}\right\rangle\right|$ is achieved 760 when the actual channel direction $\tilde{\mathbf{h}}_{1}$ is away from both the BF 761 vector $\mathbf{w}$ and the quantized channel direction $\hat{\mathbf{h}}_{1}$ and falls on 762 the quantization cell boundary. 


$$
\operatorname{SINR}_{1}=\frac{p_{11}\left\|\mathbf{h}_{1}\right\|^{2}\left(\lambda_{2 \min }\left|\xi_{5}\right|-\sqrt{\left(1-\lambda_{2 \min }^{2}\right)\left(1-\left|\xi_{5}\right|^{2}\right)}\right)^{2}}{1+p_{21}\left\|\mathbf{g}_{1}\right\|^{2}\left(\left|\lambda_{2 \min } \xi_{2}\right|+\sqrt{\left(1-\lambda_{2 \min }^{2}\right)\left(1-\left|\xi_{2}\right|^{2}\right)}\right)^{2}}
$$

Let

$$
\mathbf{g}_{1}^{\dagger}=\frac{\hat{\mathbf{g}}_{1}-\left\langle\hat{\mathbf{g}}_{1}, \hat{\mathbf{h}}_{1}\right\rangle \hat{\mathbf{h}}_{1}}{\left\|\hat{\mathbf{g}}_{1}-\left\langle\hat{\mathbf{g}}_{1}, \hat{\mathbf{h}}_{1}\right\rangle \hat{\mathbf{h}}_{1}\right\|}
$$

767 where $\mathbf{h}_{1}^{\dagger}$ is orthogonal to $\hat{\mathbf{g}}_{1}$ and can be expressed as a linear 768 combination of $\hat{\mathbf{g}}_{1}$ and $\hat{\mathbf{h}}_{1}$. Vector $\mathbf{g}_{1}^{\dagger}$ is orthogonal to $\hat{\mathbf{h}}_{1}$ and 769 can be also expressed as a linear combination of $\hat{\mathbf{g}}_{1}$ and $\hat{\mathbf{h}}_{1}$. 770 Assuming that vector $\mathbf{u}_{1}$ is an arbitrary vector with a unit norm 771 and orthogonal to both $\mathbf{h}_{1}^{\dagger}$ and $\hat{\mathbf{h}}_{1}$ and that vector $\mathbf{u}_{2}$ is an 772 arbitrary vector with a unit norm and orthogonal to both $\mathbf{g}_{1}^{\dagger}$ and $773 \hat{\mathrm{g}}_{1}$, we have

$$
\begin{aligned}
\mathbf{w} & =\xi_{1} \mathbf{h}_{1}^{\dagger}+\xi_{2} \hat{\mathbf{g}}_{1}+\xi_{3} \mathbf{u}_{1}=\xi_{4} \mathbf{g}_{1}^{\dagger}+\xi_{5} \hat{\mathbf{h}}_{1}+\xi_{6} \mathbf{u}_{2} \\
\hat{\mathbf{h}}_{1} & =\delta_{1} \mathbf{h}_{1}^{\dagger}+\delta_{2} \hat{\mathbf{g}}_{1} \\
\hat{\mathbf{g}}_{1} & =\delta_{3} \mathbf{g}_{1}^{\dagger}+\delta_{4} \hat{\mathbf{h}}_{1} .
\end{aligned}
$$

774 In the given equations, since the norms of $\mathbf{w}, \hat{\mathbf{h}}_{1}$, and $\hat{\mathbf{g}}_{1}$ are all 7751 , it follows that

$$
\begin{aligned}
& \sum_{i=1}^{3}\left|\xi_{i}\right|^{2}=\sum_{i=4}^{6}\left|\xi_{i}\right|^{2}=1 \\
& \left|\delta_{1}\right|^{2}+\left|\delta_{2}\right|^{2}=\left|\delta_{3}\right|^{2}+\left|\delta_{4}\right|^{2}=1 .
\end{aligned}
$$

776 Thus, with the aid of (40) to (44) and the two lemmas, for a 777 given set of $\hat{\mathbf{g}}_{1}$ and $\hat{\mathbf{h}}_{1}$, the minimum of $\mathrm{SINR}_{1}$ takes the form 778 of (45), shown at the top of the page. Additionally, we also have

$$
\left|\xi_{5}\right|=\left|\left\langle\mathbf{w}, \hat{\mathbf{h}}_{1}\right\rangle\right|=\left|\xi_{1} \delta_{1}^{*}+\xi_{2} \delta_{2}^{*}\right| \leq\left|\xi_{1} \delta_{1}\right|+\left|\xi_{2} \delta_{2}\right| .
$$

779 When the minimum SINR in (45) is maximized, the equality 780 on the right side of (46) holds. This is because, when $\left|\xi_{5}\right|$ 781 increases, the numerator of (45) increases, and increasing the 782 value of $\left|\xi_{5}\right|$ can be achieved by changing only the principles of $783 \xi_{1}$ and $\xi_{2}$, which will not affect the value of the denominator in 784 the equation. Additionally, we observe that, when the minimum 785 SINR in (45) is maximized, we have $\left|\xi_{3}\right|=0$. The proof 786 exploits that, if the maximum value of this $\mathrm{SINR}_{1}$ is achieved 787 when the BF vector $\mathbf{w}$ is not on the same complex plane with 788 both $\hat{\mathbf{g}}_{1}$ and $\hat{\mathbf{h}}_{1}$, we have $\left|\xi_{3} \xi_{6}\right| \neq 0$. In this case, we can hold $789\left|\xi_{2}\right|$ at a fixed value, and set $\xi_{3}$ to 0 . This will result in an 790 increase in $\left|\xi_{1}\right|$, and since

$$
\left|\xi_{5}\right|=\left|\delta_{1} \xi_{1}\right|+\left|\delta_{2} \xi_{2}\right|
$$

791 the value of $\left|\xi_{5}\right|$ increases, resulting in an increase in the value 792 of SINR $_{1}$. Upon combining (45) and (47), we arrive at (14); 793 hence, the theorem is proven.

\section{REFERENCES}

[1] M. Sawahashi, Y. Kishiyama, A. Morimoto, D. Nishikawa, and 795 M. Tanno, "Coordinated multipoint transmission/reception techniques for 796 LTE-advanced," IEEE Wireless Commun., vol. 17, no. 3, pp. 26-34, 797 Jun. 2010.

798

[2] R. Irmer, H. Droste, P. Marsch, M. Grieger, G. Fettweis, S. Brueck, 799 H. P. Mayer, L. Thiele, and V. Jungnickel, "Coordinated multipoint: Con- 800 cepts, performance, and field trial results," IEEE Commun. Mag., vol. 49, 801 no. 2, pp. 102-111, Feb. 2011.

802

[3] D. Choi, D. Lee, and J. H. Lee, "Resource allocation for CoMP with 803 multiuser MIMO-OFDMA," IEEE Trans. Veh. Technol., vol. 60, no. 9, 804 pp. 4626-4632, Nov. 2011.

805

[4] R. Bhagavatula and R. W. Heath, "Adaptive limited feedback for sum-rate 806 maximizing beamforming in cooperative multicell systems," IEEE Trans. 807 Signal Process., vol. 59, no. 2, pp. 800-811, Feb. 2011.

[5] D. Love, R. Heath, V. Lau, D. Gesbert, B. Rao, and M. Andrews, "An 809 overview of limited feedback in wireless communication systems," IEEE 810 J. Sel. Areas Commun., vol. 26, no. 8, pp. 1341-1365, Oct. 2008.

811

[6] T. Wu and V. Lau, "Design and analysis of multi-user SDMA systems 812 with noisy limited CSIT feedback," IEEE Trans. Wireless Commun, vol. 9, 813 no. 4, pp. 1446-1450, Apr. 2010.

[7] T. Wu and V. Lau, "Robust precoder adaptation for MIMO links with 815 noisy limited feedback," IEEE Trans. Inf. Theory, vol. 55, no. 4, pp. 1640- 816 1649, Apr. 2009.

[8] C. Murthy, J. Zheng, and B. Rao, "Performance of quantized equal gain 818 transmission with noisy feedback channels," IEEE Trans. Signal Process., 819 vol. 56, no. 6, pp. 2451-2460, Jun. 2008.

820

[9] A. Housfater and T. J. Lim, "Throughput of precoded broadcast transmis- 821 sion with noisy feedback," in Proc. IEEE Int. Symp. Inf. Theory, Jul. 2009, 822 pp. 398-402.

[10] A. Housfater and T. J. Lim, "Noisy feedback linear precoding: A 824 bayesian cramér-rao bound," in Proc. Int. Symp. Inf. Theory, Jul. 2009, 825 pp. 1689-1693

[11] I. S. Dhillon, R. W. Heath, T. Strohmer, and J. A. Tropp, “Constructing 827 packings in Grassmannian manifolds via alternating projection," Exp. 828 Math., vol. 17, no. 7, pp. 9-35, 2008.

[12] Y. Cheng, V. Lau, and Y. Long, "A scalable limited feedback design for 830 network MIMO using per-cell product codebook," IEEE Trans. Wireless 831 Commun., vol. 9, no. 10, pp. 3093-3099, Oct. 2010.

[13] X. Hou and C. Yang, "Codebook design and selection for multi-cell 833 cooperative transmission limited feedback systems," in Proc. IEEE 73rd 834 VTC, May 2011, pp. 1-5.

[14] D. Su, X. Hou, and C. Yang, "Quantization based on per-cell codebook 836 in cooperative multi-cell systems," in Proc. IEEE WCNC, Mar. 2011, 837 pp. 1753-1758.

838

[15] X. Hou and C. Yang, "How much feedback overhead is required for base 839 station cooperative transmission to outperform non-cooperative transmis- 840 sion?" in Proc. IEEE ICASSP, May 2011, pp. 3416-3419.

[16] W. Yu, T. Kwon, and C. Shin, "Multicell coordination via joint scheduling, 842 beamforming and power spectrum adaptation," in Proc. IEEE INFOCOM, 843 Apr. 2011, pp. 2570-2578.

[17] P. Viswanath, D. Tse, and R. Laroia, "Opportunistic beamforming using 845 dumb antennas," IEEE Trans. Inf. Theory, vol. 48, no. 6, pp. 1277-1294, 846 Jun. 2002.

[18] D. Park and G. Caire, "Hard fairness versus proportional fairness in wire- 848 less communications: The multiple-cell case," in Proc. IEEE Int. Symp. 849 Inf. Theory, Jul. 2008, pp. 2036-2040.

[19] S. A. Vorobyov, A. B. Gershman, and Z.-Q. Luo, "Robust adaptive beam- 851 forming using worst-case performance optimization: a solution to the 852 signal mismatch problem," IEEE Trans. Signal Process., vol. 51, no. 2, 853 pp. 313-324, Feb. 2003.

[20] R. Lorenz and S. Boyd, "Robust minimum variance beamforming," IEEE 855 Trans. Signal Process, vol. 53, no. 5, pp. 1684-1696, May 2005.

[21] A. Abdel-Samad, A. B. Gershman, and T. N. Davidson, "Robust transmit 857 beamforming based on imperfect channel feedback," in Proc. IEEE 60th 858 VTC-Fall, Sep. 2004, vol. 3, pp. 2049-2053. 
860 [22] E. Gharavol, Y.-C. Liang, and K. Mouthaan, "Robust downlink beam861 forming in multiuser MISO cognitive radio networks with imperfect 862 channel-state information," IEEE Trans. Veh. Technol., vol. 59, no. 6, 863 pp. 2852-2860, Jul. 2010.

864 [23] W. Santipach and M. Honig, "Asymptotic capacity of beamforming with 865 limited feedback," in Proc. ISIT, Jul. 2004, p. 290.

866 [24] T. Yoo, N. Jindal, and A. Goldsmith, "Multi-antenna downlink channels 867 with limited feedback and user selection," IEEE J. Sel. Areas Commun., 868 vol. 25 , no. 7, pp. 1478-1491, Sep. 2007.

869 [25] K. K. Mukkavilli, A. Sabharwal, E. Erkip, and B. Aazhang, "On beam870 forming with finite rate feedback in multiple-antenna systems," IEEE 871 Trans. Inf. Theory, vol. 49, no. 10, pp. 2562-2579, Oct. 2003.

872 [26] G. Marsaglia, "Choosing a point from the surface of a sphere," Ann. Math. 873

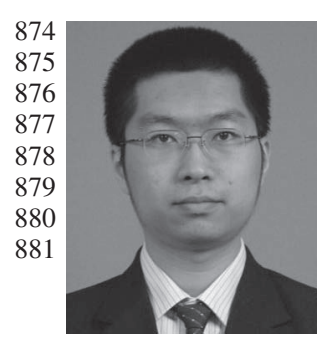

Yingxiang Yang received the B.E. degree from Tsinghua University, Beijing, China, in 2012. He is currently working toward the Ph.D. degree with Rutgers University, New Brunswick, NJ, USA.

His research interests include communications theory, optimization theory, and game theory.

Mr. Yang has been a Peer Reviewer for the IEEE Vehicular Technology Conference since 2011.

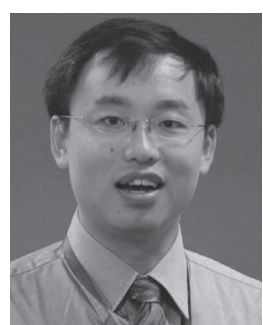

Wei Chen (SM'13) received the B.S. and Ph.D. 908 degrees in electronic engineering (first-class honors) 909 from Tsinghua University, Beijing, China, in 2002, 910 and 2007, respectively.

From 2005 to 2007, he was a Visiting Research 912 Staff with the Hong Kong University of Science and 913 Technology, Hong Kong. Since July 2007, he has 914 been with the Department of Electronic Engineer- 915 ing, Tsinghua University, where he is currently a 916 Full Professor and the Vice-Director of the Institute 917 of Communications. From June 2010 to September 918 2010, he was a Visiting Researcher with the University of Southampton, 919 Southampton, U.K. He is a 973 Youth Project Chief Scientist and is supported 920 by the New Century Talent Program of the Chinese Ministry of Education, 921 the Beijing Nova Program, and 100 fundamental research talents program of 922 Tsinghua University (also known as the 221 Talents Program). His research 923 interests include wireless communications, information theory, and applied 924 optimization.

925

Dr. Chen served as a Vice-Director of the Youth Committee of the China 926 Institute of Communications, a Tutorial Cochair of the 2013 IEEE International 927 Conference on Communications, a track Cochair of the Wireless Track of 928 the IEEE Consumer Communications and Networking Conference in 2013, a 929 Cochair of the Technical Program Committee (TPC) of the IEEE Vehicular 930 Technology Conference in Spring 2011, the Publication Chair of the IEEE 931 International Conference on Communications in China in 2012, a TPC Cochair 932 of the Wireless Communication Symposium at the 2010 IEEE International 933 Conference on Communications (ICC), and a Student Travel Grant Chair 934 of ICC 2008. He also served as an Editor for IEEE WiRELESS COMMU- 935 NICATIONS LETTERS. He received the 2010 IEEE Communication Society 936 Asia-Pacific Board Best Young Researcher Award; the 2009 IEEE Marconi 937 Prize Paper Award; the Best Paper Awards at the IEEE ICC in 2006, the 938 First IEEE International Workshop on Cross Layer Design in 2007, and the 939 IEEE Smart Gird Commmunications Conference in 2012; the 2011 Tsinghua 940 Rising Academic Star Award; the 2012 Tsinghua Young Faculty Teaching 941 Excellence Award; the First Prize at the First National Young Faculty Teach- 942 ing Competition; the First Prize at the Seventh Beijing Young Faculty Tea- 943 ching Competition; the First Prize at the Fifth Tsinghua University Young 944 Faculty Teaching Competition; and the National May 1st Labor Medal.

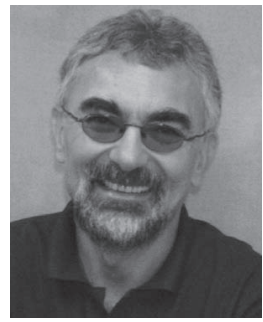

Lajos Hanzo (M'91-SM'92-F'04) received the 946 M.S. degree (with first-class honors) in electronics 947 and the Ph.D. degree from the Technical University 948 of Budapest, Budapest, Hungary, in 1976 and 1983, 949 respectively, the D.Sc. degree from the University of 950 Southampton, Southampton, U.K., in 2004, and the 951 "Doctor Honoris Causa" degree from the Technical 952 University of Budapest in 2009.

During his 35-year career in telecommunications, 954 he has held various research and academic posts in 955 Hungary, Germany, and the U.K. Since 1986, he has 956 been with the School of Electronics and Computer Science, University of 957 Southampton, Southampton, U.K., where he holds the Chair for Telecommuni- 958 cations. Since 2009, he has been a Chaired Professor with Tsinghua University, 959 Beijing China. He is currently directing a 100-strong academic research team, 960 working on a range of research projects in the field of wireless multimedia 961 communications sponsored by industry; the Engineering and Physical Sciences 962 Research Council, U.K.; the European IST Programme; and the Mobile Virtual 963 Centre of Excellence, U.K. He is an enthusiastic supporter of industrial and 964 academic liaison and offers a range of industrial courses. He has successfully 965 supervised $80 \mathrm{Ph} . D$. students, coauthored 20 John Wiley/IEEE Press books on 966 mobile radio communications totaling in excess of 10000 pages, published 967 more than 1250 research entries on IEEE Xplore, and presented keynote 968 lectures. (For further information on research in progress and associated 969 publications, please refer to http://www-mobile.ecs.soton.ac.uk.)

Dr. Hanzo is Fellow of the Royal Academy of Engineering, U.K., a Fellow 971 of the Institution of Electrical Engineers, and a Governor of the IEEE Vehicular 972 Technology Society. He has been a Technical Program Committee Chair and a 973 General Chair for IEEE conferences. During 2008-2012, he was the Editor-in- 974 Chief of the IEEE Press. He has received a number of distinctions.
906 Electronic Engineering Award from Tsinghua University and the Outstanding 907 Graduates of Shaanxi Province Award. 


\section{AUTHOR QUERIES}

AUTHOR PLEASE ANSWER ALL QUERIES

AQ1 = Please provide keywords

END OF ALL QUERIES 


\title{
A Low-Complexity Cross-Layer Algorithm for Coordinated Downlink Scheduling and Robust Beamforming Under a Limited Feedback Constraint
}

\author{
Yingxiang Yang, Bo Bai, Member, IEEE, Wei Chen, Senior Member, IEEE, and Lajos Hanzo, Fellow, IEEE
}

5 Abstract-Coordinated scheduling/beamforming (CS/CB) sub6 stantially mitigates the intercell interference (ICI), hence increas7 ing the cell-edge throughput on the downlink (DL) of coordinated 8 multipoint (CoMP) systems. To maximize the DL throughput, the 9 cooperating base stations (BSs) jointly select the best set of users 10 for DL scheduling and then jointly design a set of beamforming 11 (BF) vectors to approach the throughput limit. However, finding 12 the optimal BF vectors requires an exhaustive search and sub13 stantial channel state information (CSI) feedback, hence resulting 14 in high algorithmic complexity and heavy uplink traffic load. 15 Hence, we conceive a new cross-layer algorithm to achieve high 16 performance at a lower feedback amount and at lower algorithmic 17 complexity. Based on the fact that different BSs usually have 18 different traffic loads, we divide the BSs into two different types, 19 i.e., the master BSs (MBSs) and the slave BSs (SBSs), where MBSs 20 have a higher transmission priority than SBSs. The scheduling 21 relies on an interference threshold, whereas our robust BF scheme 22 exploits both the channel direction information (CDI), which is 23 quantized using the technique of limited feedback, and the channel 24 quality information (CQI), which is assumed to be fed back accu25 rately. Our numerical results show that the proposed algorithm 26 does not lose much performance compared with that achieved by 27 an exhaustive search, whereas the algorithmic complexity is as low 28 as that of the solutions operating without CoMP.

29 Index Terms-Author, please supply index terms/keywords 30 for your paper. To download the IEEE Taxonomy go to 31 http://www.ieee.org/documents/taxonomy_v101.pdf .

\section{INTRODUCTION}

33

34 OORDINATED multipoint (CoMP) transmission is a key feature in the Long-Term Evolution system [1]-[3], which 35 promises performance improvements for the cell-edge users by 36 allowing several base stations (BSs) to cooperate. On the uplink

Manuscript received August 7, 2012; revised February 5, 2013 and May 17, 2013; accepted June 22, 2013. This paper was supported in part by the National Basic Research Program of China (973 Program) under Grant 2013CB336600 and Grant 2012CB316000, by the U.K.-China Bridge Fellowship, by Chuanxin Funding, by the Beijing Nova Program, and by the Chinese Ministry of Education New Century Talent Program. The work of L. Hanzo was supported by the European Research Council under their Advanced Fellow Grant. The review of this paper was coordinated by Dr. J.-C. Chen.

Y. Yang was with Tsinghua University, Beijing 100084, China. He is currently with the Department of Electrical and Computer Engineering, Rutgers University, New Brunswick, NJ 08901 USA (e-mail: yangyx.thu @ gmail.com).

B. Bai and W. Chen are with the Department of Electronic Engineering, Tsinghua University, Beijing, 100084, China (e-mail:eebobai@tsinghua. edu.cn; wchen@tsinghua.edu.cn).

L. Hanzo is with the School of Electronic and Computer Science, University of Southampton, Southampton, SO17 1BJ U.K. (e-mail: 1h@ecs.soton.ac.uk).

Color versions of one or more of the figures in this paper are available online at http://ieeexplore.iee.org.

Digital Object Identifier 10.1109/TVT.2013.2271876 side, the cooperating BSs share the information and jointly pro- 37 cess the data received from the mobile stations (MSs). On the 38 downlink (DL) side, two commonly implemented methods are 39 the joint processing and coordinated scheduling/beamforming 40 (CS/CB) schemes, where CS/CB allows the BSs to coopera- 41 tively schedule their DL transmissions to a set of users and 42 then cooperatively design a set of efficient beamforming (BF) 43 vectors. Under the assumption that the users' channel state 44 information (CSI) is perfectly known at the BSs' DL trans- 45 mitters, the throughput performance of coordinated BF varies 46 for different sets of scheduled users. Thus, the ultimate task for 47 the BSs is to schedule their DL transmissions to the optimal 48 set of users that are capable of approaching the maximum DL- 49 transmission throughput and then to design the particular set of 50 $\mathrm{BF}$ vectors that can approach this limit.

However, the problem described earlier has the following 52 obstacles.

1) High algorithmic complexity. The algorithmic complex- 54 ity imposed by finding the optimal set of MSs for which 55 the DL transmissions should be scheduled is high since 56 an exhaustive search is required for optimal scheduling. 57 Assuming that there are $M$ BSs and that the user set of 58 the $i$ th $\mathrm{BS}(\mathrm{BS} i)$ is denoted by $\mathcal{U}_{i}$, the complexity of the 59 scheduling algorithm is on the order of $\mathcal{O}\left(\prod_{i=1}^{M}\left|\mathcal{U}_{i}\right|\right) .60$ This complexity becomes excessive, when the number of 61 BSs and MSs in each cell increases.

2) High feedback load. Assuming that the feedback "bud- 63 get" of each MS's CSI is $B$ bits for the Channel Di- 64 rection Information (CDI) and $b$ bits for the Channel 65 Quality Information (CQI), the feedback traffic load can 66 be expressed as $\left(\sum_{i=1}^{M}\left|\mathcal{U}_{i}\right|\right)(M B+b)$, where the $M B 67$ bits of the CDI feedback are related to $M$ channels, i.e., 68 one for the specific channel receiving the desired signal 69 and the remaining $(M-1)$ for the channel receiving the 70 interfering signal.

3) High backhaul traffic. To calculate the set of optimal BF 72 vectors, at least one of the BSs has to know the CSI of 73 all the MSs. Thus, the backhaul traffic load is at least 74 $\left(\sum_{i=1}^{M}\left|\mathcal{U}_{i}\right|-\max _{i}\left\{\left|\mathcal{U}_{i}\right|\right\}\right)(M B+b)$.

4) Inaccuracy caused by imperfect CSI feedback. Since the 76 CSI feedback introduces both quantization errors and 77 latency, the CSI acquired at the BSs is inaccurate. Thus, 78 it is possible that the DL-scheduling decision will be 79 inaccurate when the quantization error is high. 
81 There has been a plethora of contributions related to CoMP 82 [1], [4]-[15], exploring possible solutions and finding remedies 83 to the impediments aforementioned. Although the original con84 tributions relied on the assumption of perfect CSI [4], more 85 realistic recent contributions assumed imperfect CSI feedback, 86 where the channel vectors are quantized to a codeword stored 87 in a codebook designed offline and the index of the code88 word is fed back instead of the actual quantized values of the 89 channel vectors. Hence, the amount of feedback per user can 90 be substantially reduced. A comprehensive introduction to the 91 topic of limited feedback aid communications can be found 92 in [5], where the authors discussed the feedback design in a 93 broad range of scenarios, employing methods used in industrial 94 standards and protocols.

95 One of the common issues in realistic limited-feedback-aided 96 systems is the inaccuracy of the CSI feedback both due to the 97 delay encountered and by the transmission errors imposed by 98 the feedback channels [6]-[10]. In [6], Wu and Lau proposed 99 a feedback design for spatial-division multiple-access (SDMA) 100 systems, demonstrating that their scheme is robust against feed101 back channel errors and characterized the system's goodput. 102 Another contribution of $\mathrm{Wu}$ and Lau [7] provided two robust 103 designs for multiple-input-multiple-output precoder adaptation 104 under the scenario of potentially error-prone limited feedback 105 and showed that both frameworks provided significant gains 106 compared with the idealized designs assuming no feedback 107 errors. In [8] and [9], the performances of equal gain trans108 mission and precoded broadcast transmission were studied, 109 respectively, under the scenario of error-prone limited feedback. 110 Finally, in [10], Housfater and Lim derived a Cramér-Rao-type 111 lower bound for linear precoders. These contributions provided 112 insights into the mitigation of the detrimental impact of the 113 imperfect CSI feedback channel.

114 Another common issue that arises when limited feedback is 115 applied to a typical CoMP system is the codebook design prob116 lem. Although the design of codebooks conceived for limited117 feedback-aided systems has been extensively studied [1], [11], 118 the number of BSs in a CoMP cluster may vary over time, hence 119 requiring a specific design. Thus, it is a challenge to design a 120 codebook imposing low overhead when the number of coop121 erating BSs is high. A promising solution is based on the per122 cell codebook design philosophy of [12]-[15], which separately 123 quantizes the channel associated with each cell within a CoMP 124 channel matrix to avoid a large codebook and to circumvent 125 frequent updates of the codebook owing to either user mobility 126 or due to the different clustering of the BSs. To elaborate a little 127 further, Cheng et al. [12] presented a limited-feedback-based 128 per-cell codebook design and showed that its performance is 129 close to that of the conventional joint-cell codebook design 130 having high overhead. In [13], attention is focussed on the 131 problem of optimal per-cell codebook designs and derived a 132 closed-form solution for the codebook size that minimizes the 133 quantization error on average. In [14], a method of reconstruct134 ing the CoMP channel's CDI was first proposed and then, the 135 performance of different codebook generation techniques and 136 per-cell codeword selection methods was compared.

137 In contrast with the insightful contributions listed earlier, we 138 pursue a different approach in reducing both the algorithmic complexity and the CSI feedback overhead for a scenario where 139 $\mathrm{CS} / \mathrm{CB}$ is employed.

1) We conceive a low-complexity noniterative cross-layer 141 algorithm, which is based on the fact that, in multicell 142 systems, all BSs tend to have different DL-transmission 143 rate requirements and traffic loads. We commence by 144 classifying the BSs into two types. The BSs having higher 145 transmission rate requirements are referred to as master 146 BSs (MBSs), which benefit from a higher priority. The re- 147 maining BSs having lower transmission rate requirements 148 are referred to as slave BSs (SBSs), which have a lower 149 priority.

150

2) We propose a low-complexity interference-threshold- 151 based algorithm for scheduling, which is combined with 152 appropriately adjusting the BF vectors of the cooperating 153 BSs. As shown in Section III, this part of the algorithm 154 only relies on the CSI at the user's side; thus, it is 155 capable of effectively reducing the CSI feedback load 156 while mitigating the inaccuracy of CSI feedback imposed 157 by the error-prone feedback channel.

158

3) Furthermore, we propose a new robust BF vector design 159 for the scenario, where the CDI and CQI are fed back sep- 160 arately. More explicitly, the CDI is quantized before being 161 fed back, whereas the CQI is assumed to be perfectly fed 162 back to the BS.

163

4) We will demonstrate both with the aid of our theoretical 164 derivation and by numerical simulations that our algo- 165 rithm has similar algorithmic complexity as the nonco- 166 operative algorithms. It imposes low backhaul traffic and 167 circumvents the dynamic channel-matrix clustering of 168 CoMP.

5) We also show that the performance of the proposed algo- 170 rithm is not overly compromised and that its performance 171 is similar to that of the iterative algorithm proposed in 172 [16], as far as the MBS is concerned. Our algorithmic 173 philosophy is highlighted in a simple scenario, where 174 only two BSs are involved, but it may be readily ex- 175 tended to more general scenarios supporting multiple BSs 176 without a dramatic increase in complexity and feedback 177 requirements.

The remainder of this paper is organized as follows. In 179 Section II, our system model is introduced, along with some 180 of our basic assumptions. In Section III, both the proposed 181 scheduling and our BF algorithm are detailed. In Section IV, 182 we present a comparison of the algorithmic complexity and the 183 signaling overhead of different algorithms. Our performance 184 analysis is provided in Section V, whereas Section VI offers 185 our conclusions. The proof of some of the theorems is provided 186 in the Appendix.

\section{SySTEM MODEL}

Consider the two-cell network of Fig. 1, where each cell has 189 a BS at its center and multiple MSs scattered within the cell. 190 BS1 on the left of Fig.1 is assumed to be the MBS, and BS2 191 on the right is an SBS. Both BS1 and BS2 are equipped with 192 $K$ transmit antennas, whereas each MS has a single receive 193 antenna. We assume that, each time, each BS schedules its DL 194 


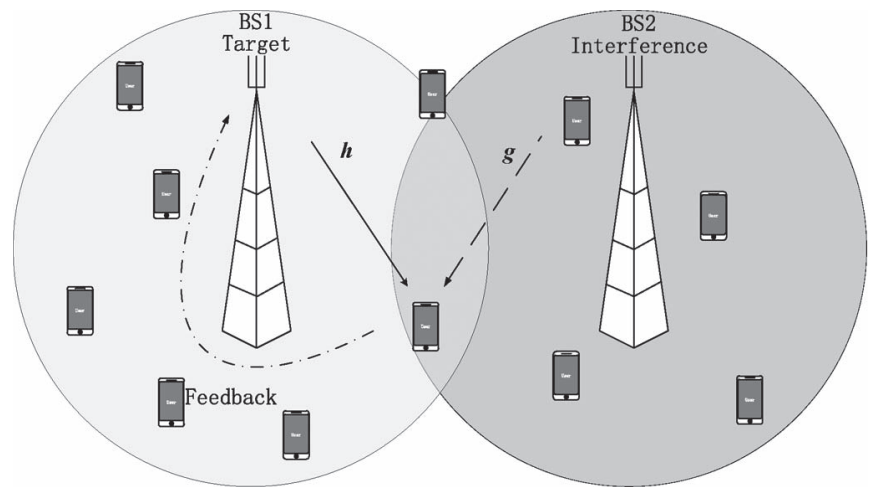

Fig. 1. System model.

195 transmission to a single MS within its cell, and different BSs 196 use the same frequency; hence, the MSs suffer from intercell 197 interference (ICI) imposed by the neighboring BS. The received 198 signal power is related to the location of the MS. There are a 199 number of studies on the fairness control issues of scheduling 200 algorithms [17], [18], but in this paper, we focus our attention 201 on the scenario where the MSs are located at the cell edge, and 202 we assume that all the MSs have the same large-scale fading 203 factor. In other words, the fairness effect of different large-scale 204 fading factors is not considered here.

205 Again, we denote the user set of cell $i(i \in\{1,2\})$ as $\mathcal{U}_{i}$. 206 Then, the signal received by user $j\left(j \in\left\{1,2, \ldots,\left|\mathcal{U}_{1}\right|\right\}\right)$ in cell 2071 and user $k\left(k \in\left\{1,2, \ldots,\left|\mathcal{U}_{2}\right|\right\}\right)$ in cell 2 can be expressed as

$$
\left\{\begin{array}{l}
y_{1, j}=p_{11, j}\left\langle\mathbf{w}_{1}, \mathbf{h}_{1, j}\right\rangle u_{1, j}+p_{21, j}\left\langle\mathbf{w}_{2}, \mathbf{g}_{1, j}\right\rangle u_{2, k}+n_{j} \\
y_{2, k}=p_{22, k}\left\langle\mathbf{w}_{2}, \mathbf{h}_{2, k}\right\rangle u_{2, k}+p_{12, k}\left\langle\mathbf{w}_{1}, \mathbf{g}_{2, k}\right\rangle u_{1, j}+n_{k}
\end{array}\right.
$$

208 where $\langle\mathbf{x}, \mathbf{y}\rangle$ represents the inner product of the vectors $\mathbf{x}$ and $209 \mathbf{y}$. Variable $y_{i, j}$ represents the signal received by user $j$ in 210 cell $i$, where $i$ equals either 1 or 2 . At the receiver of the $j$ th 211 user in cell $i_{1}$, the signal strength received from cell $i_{2}$ can be 212 represented as $p_{i_{1} i_{2}}, j$. The power of the symbol transmitted 213 from BS $i$ to its $j$ th user is denoted $u_{i, j}$, which is normalized 214 as $E\left\{\left|u_{i, j}\right|^{2}\right\}=1$. The random variables $n_{j}$ and $n_{k}$ represent 215 the normalized Gaussian noise, with $E\left\{\left|n_{k}\right|^{2}\right\}=E\left\{\left|n_{j}\right|^{2}\right\}=$ 2161 , whereas vector $\mathbf{h}_{i, j} \in \mathbb{C}^{K \times 1}$ represents the DL channel 217 conditions between $\mathrm{BS} i$ and its $j$ th user, which can be viewed as 218 the target channel condition. Furthermore, vector $\mathbf{g}_{i, j} \in \mathbb{C}^{K \times 1}$ 219 denotes the DL channel condition between the $j$ th user of $220 \mathrm{BS} i$ and the neighboring $\mathrm{BS}$, which is the interferring channel. 221 The target channel vectors and the interfering channel vectors 222 are independent and identically distributed in terms of their 223 statistics, and they both follow a probability distribution of $224 C N\left(0, \mathbf{I}_{K}\right)$. Finally, vector $\mathbf{w}_{i}$ is the BF vector adopted by BSi. 225 The goal of the proposed algorithm is to increase the 226 DL throughput, which is quantified in terms of the channel 227 capacity of

$$
R_{i}=\log \left(1+\operatorname{SINR}_{i}\right)
$$

228 where $\operatorname{SINR}_{i}$ is the signal-to-interference-plus-noise ratio at 229 the scheduled user's terminal of BSi. We assume that each 230 BS schedules its DL transmission to a single MS each time. 231 Thus, to simplify the notation, we denote the target channel condition and the interfering channel condition of the scheduled 232 MS located in cell $i$ by $\mathbf{h}_{i}$ and $\mathbf{g}_{i}$, respectively, yielding

$$
\operatorname{SINR}_{i}=\frac{p_{i i}\left\|\mathbf{h}_{i}\right\|^{2}\left|\left\langle\mathbf{w}_{i}, \tilde{\mathbf{h}}_{i}\right\rangle\right|^{2}}{1+p_{j i}\left\|\mathbf{g}_{i}\right\|^{2}\left|\left\langle\mathbf{w}_{j}, \tilde{\mathbf{g}}_{i}\right\rangle\right|^{2}} .
$$

Note that, in the given equation, the norms of vectors $\mathbf{h}_{i}$ and 234 $\mathrm{g}_{i}$ are separated from their directions so that we have $\left\|\tilde{\mathbf{h}}_{i}\right\|=235$ $\left\|\tilde{\mathbf{g}}_{i}\right\|=1$. We use $p_{i i}$ to denote the signal strength received by 236 the selected user in cell $i$ and $p_{j i}$ to denote the strength of the 237 interfering signal arriving from cell $j$ contaminating the desired 238 user's signal in cell $i$. We simplified the subscript since we only 239 consider the case where each BS schedules its DL transmission 240 to a single user at a time.

\section{A. Schemes Operating Without CoMP}

242

Transmission schemes operating without CoMP typically 243 have lower complexity than those relying on CoMP. Here, 244 we simply consider the classic time-division multiple-access 245 (TDMA) and frequency-division multiple-access (FDMA) 246 schemes. In the TDMA scheme, the $M$ BSs transmit sequen- 247 tially so that each BS transmits in $1 / M$ fraction of the time, 248 without imposing any ICI. In the FDMA scheme, on the other 249 hand, the $M$ BSs share the transmission bandwidth so that each 250 BS transmits in a separate subband without being interfered by 251 the neighboring BSs.

We compare the performance of the TDMA and FDMA 253 schemes to that of our proposed algorithm and to the exhaustive 254 search algorithm. In TDMA and FDMA schemes, all the MSs 255 feed back both their CQI and their target channel conditions 256 using limited feedback, so that the BSs can design BF vectors 257 accordingly. The BSs, on the other hand, schedule their DL 258 transmission for the specific MS having the highest CQI. The 259 transmission throughput for BS $i$ can be expressed as

$$
R_{i}=\frac{1}{M} \log \left(1+\max _{j \in \mathcal{U}_{i}} \operatorname{SNR}_{j}\right)
$$

where $M=2$ in our scenario.

\section{B. Iterative Scheduling and Beamforming}

Iterative algorithms are capable of reducing the algorith- 263 mic complexity while maintaining a similar performance to 264 their exhaustive-search-algorithm-based counterparts. In this 265 paper, we adopt the iterative approach proposed in [16] as a 266 benchmark for our performance evaluation, which relies on the 267 following three steps.

1) Fix the power allocation and scheduled users; then, find 269 the best combination of BF vectors.

2) Fix the BF vectors and power allocation; then, find the 271 best set of users for scheduling.

3) Fix the BF vectors and the scheduled users; then, update 273 the power allocation among the scheduled users.

Since the scenario that we study assumes fixed power allo- 275 cation, the given three-step algorithm reduces to two steps in 276 


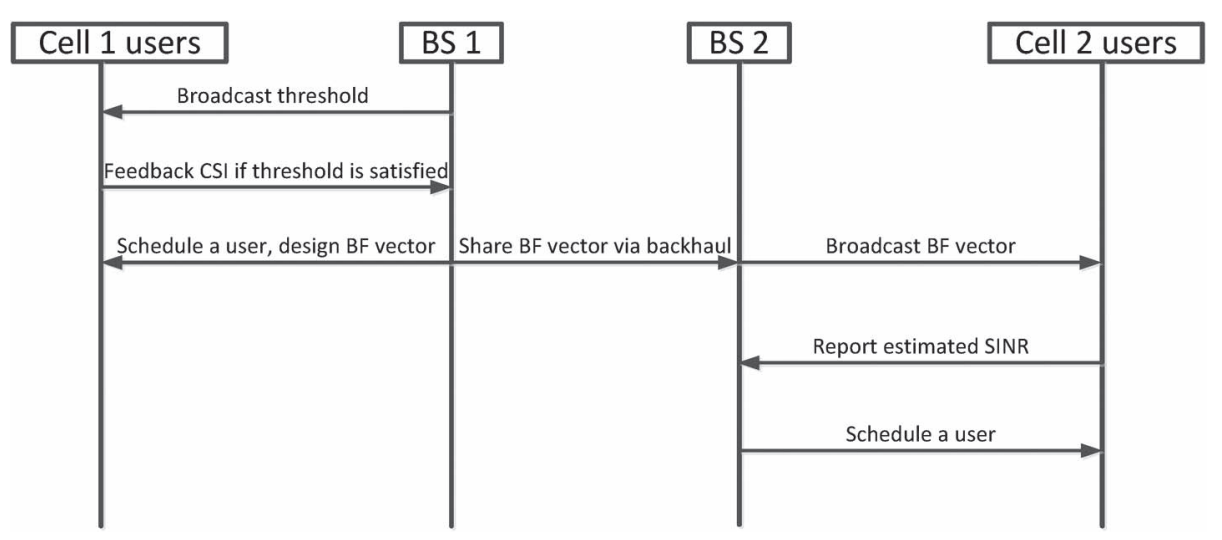

Fig. 2. Algorithm outline.

277 which the combination of BF vectors and the set of scheduled 278 users is updated in an iterative fashion as follows.

1) Fix the set of scheduled users; then, find the optimal set of BF vectors correspondingly.

2) Fix the set of BF vectors; then, find the set of users that yield optimal throughput performance correspondingly.

283 The performance of the algorithm is characterized in 284 Section V. LIMITED FEEDBACK CONSTRAINT

287 The algorithmic steps are shown in Fig. 2. In the first step, 288 the MBS, i.e., BS1, broadcasts a threshold value constraining 289 the channel directions of the desired users with respect to the 290 interfering BS to maintain the target integrity. Explicitly, the 291 directions of the desired user's target channel and of the same 292 user's interfering channel should be perpendicular to each other, 293 which corresponds to the absolute value of their inner product 294 being close to zero. The users within cell 1 receive the threshold 295 value and decide whether or not to feed their CSI back to their 296 anchor BS. If a desired user's target channel and interfering 297 channel satisfy the required angular separation threshold con298 straint, he/she feeds back the CDI of both the target channel and 299 the interfering channel, as well as the CQI of both channels. 300 Here, the CQI is defined as the product of $p_{i i}\left\|\mathbf{h}_{i}\right\|^{2}$ for the 301 target channel and $p_{j i}\left\|\mathbf{g}_{i}\right\|^{2}$ for the interfering channel, when 302 the user is located in cell $i$. Once the CSI of all the users that 303 satisfy the angular separation constraint has been fed back to the $304 \mathrm{MBS}$, the MBS decides which particular user to schedule for its $305 \mathrm{DL}$ transmission and then designs the BF vector, following our 306 robust $\mathrm{BF}$ vector design method to be presented later. Once the $307 \mathrm{BF}$ vector is determined, it is shared with the SBS, i.e., BS2, 308 via a backhaul link, the SBS broadcasts the BF vector, and all 309 the users within cell 2 feed back their expected SINR values 310 computed with the aid of the BF vector and their local CSI. In 311 the final step, the SBS schedules its DL transmissions to the 312 user having the highest "reported" SINR value.

313 The algorithm introduced relies on a few important assump314 tions, which are based on the following motivation.

315 1) Introduction of the constraint $\mathbf{w}_{1}=\mathrm{w}_{2}$. In the original 316 $\mathrm{CS} / \mathrm{CB}$ problem, the $\mathrm{BF}$ vectors of different BSs do not necessarily have the same direction. In fact, if not shared 317 via the backhaul link, the BF vector of a BS can be 318 regarded as a random vector both for the other BSs and for 319 all the MSs of the neighboring cells. There is no simple 320 yet elegant way of effectively reducing the size of the 321 candidate user set, but introducing the given constraint 322 brings us obvious benefits. First, when we have $\mathbf{w}_{1}=323$ $\mathbf{w}_{2}$, the local CSI can be directly used to compute the 324 level of interference, which is now $\left\langle\tilde{\mathbf{h}}_{1}, \tilde{\mathbf{g}}_{2}\right\rangle$ for cell 1 and 325 $\left\langle\tilde{\mathbf{h}}_{2}, \tilde{\mathbf{g}}_{1}\right\rangle$ for cell 2 . This can assists us in exploiting the 326 CSI at the MSs for naturally ruling out DL transmission 327 to the MSs suffering from severe interference. As will be 328 shown in Section IV, the feedback load is substantially 329 reduced. Second, both the scheduling and BF parts of 330 the algorithm can be implemented in each cell by relying 331 merely on local CSI, which means that the backhaul traf- 332 fic is effectively reduced. Additionally, the algorithmic 333 complexity is also significantly cut down since only a 334 small portion of the MBS's users perform CSI feedback, 335 whereas the users of the SBS only feed their CQI back to 336 the BS. These complexity and feedback requirements are 337 similar to or even lower than those of some standard non- 338 CoMP solutions, such as those of the TDMA and FDMA 339 schemes.

2) Introduction of the MBS and the SBS. The related assump- 341 tions are based on the fact that, at each moment, it is 342 likely that some BSs have a higher transmission rate re- 343 quirement than the others; hence, they should be granted 344 a higher priority and, ultimately, a higher transmission 345 rate. Hence, an MBS can schedule its DL transmission 346 to a user and design the BF vector with a higher priority, 347 whereas the SBS cannot. The performance loss imposed 348 by this unbalanced priority can be partially recovered 349 when the number of users in the cells is high.

\section{A. Threshold-Based User Scheduling}

As shown in Fig. 2, the BSs schedule their DL transmissions 352 according to a thresholding algorithm based on a carefully 353 designed threshold. In our proposed algorithm, the MBS and 354 the SBS have the same BF directions. Here, we focus on 355 scheduling the DL transmissions to the specific MS, whose tar- 356 get channel direction $\tilde{\mathbf{h}}$ is "most different" from its interfering 357 


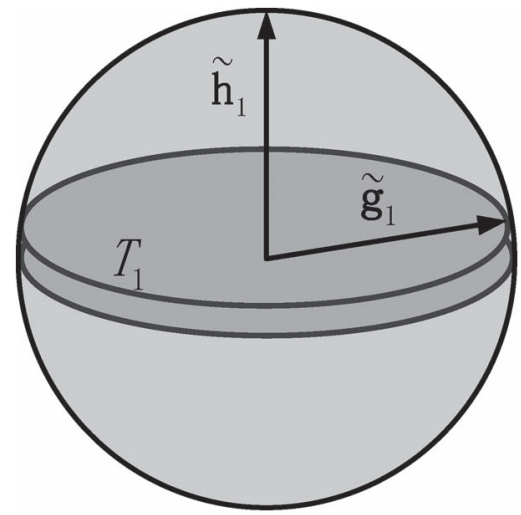

Fig. 3. Threshold.

358 channel direction $\tilde{\mathrm{g}}$ among all the users. More explicitly, we 359 have to find the specific user, whose target channel direction and 360 interfering channel direction have the smallest inner product 361 absolute value across the entire set of users served by the MBS. 362 However, it should be noted that the user to be scheduled for DL 363 transmission in CoMP relying on an exhaustive search might 364 not have an $\tilde{\mathbf{h}}$ perpendicular to $\tilde{\mathrm{g}}$. The thresholds can be defined 365 as follows.

366 The MBS schedules the user whose channel conditions 367 satisfy

$$
\left|\left\langle\tilde{\mathbf{h}}_{1}, \tilde{\mathbf{g}}_{1}\right\rangle\right| \leq T_{1}
$$

368 where $T_{1}$ is the relevant threshold. An intuitive interpretation 369 of the threshold is shown in Fig. 3, which shows (5) with the 370 aid of $T_{1}$. Since the norms of both the BF vectors and of the 371 channel directions of the MSs are 1, these vectors can be placed 372 on a globe-like unit-radius hypersphere in $\mathbb{C}^{K}$, with one end at 373 the origin and the other on the surface of the hypersphere. By 374 assuming that the target channel direction $\tilde{\mathbf{h}}_{1}$ of the scheduled 375 user points to the "north pole" of the globe, the interfering 376 channel direction $\tilde{\mathrm{g}}_{1}$ will fall within the area bounded by the 377 "Tropic," which is characterized by the value of $T_{1}$.

378 Intuitively, when the threshold $T_{1}$ becomes looser, i.e., when 379 it approaches 1, more users will satisfy (5), and the complexity 380 of the algorithm is increased. In particular, when we have $T_{1}=$ 381 1, all the users feed back their CSI, and the algorithm becomes 382 identical to the exhaustive search for the MBS. By contrast, 383 when $T_{1}$ approaches 0 , the scheduling part of the algorithm will 384 guarantee a higher transmission rate for the scheduled users, 385 but it also comes more likely that no users satisfy (5), which 386 leads to lower algorithmic complexity and reduced feedback 387 load. Thus, the threshold controls the tradeoff between the 388 algorithmic complexity and the desired performance; hence, 389 it should be determined under the constraint of ensuring a 390 minimum probability of at least one successful DL scheduling 391 for the entire set of users. The selection of the threshold based 392 upon the given principle can be achieved with the aid of the 393 following theorem.

394 Theorem 1: Let us assume that there are $N_{1}$ users in cell 3951 . Then, for the MBS, the probability of a successful DL 396 scheduling action can be expressed as

$$
P_{\text {suc1 }}=1-\left(1-T_{1}^{2}\right)^{N_{1}(K-1)} .
$$

Proof: See Appendix A.

The performance achieved with the aid of this threshold is 398 characterized in Section IV.

B. Robust Beamforming Under Limited Feedback for the Channel Direction Information

According to Fig. 2, upon scheduling the DL transmission to 402 a user whose channel conditions satisfy (5), the MBS adopts a 403 $\mathrm{BF}$ vector that further improves the throughput. Since the MSs 404 perform limited feedback of their channel conditions, when 405 quantizing both $\tilde{\mathbf{h}}_{1}$ and $\tilde{\mathbf{g}}_{1}$ using a preset codebook and when 406 transmitting the index of a codeword, the quantization error im- 407 poses inaccuracy on the design of BF vectors. We mitigate this 408 impact using a robust BF technique, which maximizes the low- 409 est possible SINR of the specific user selected. Numerous stud- 410 ies have been dedicated to robust BF [19]-[22]. Although the 411 scenarios of these contributions are different, they all model the 412 quantization error as an additive noise vector. For example, 413 the target channel's channel vector of the selected user in cell 1414 would be modeled as

$$
\tilde{\mathbf{h}}_{1}=\hat{\mathbf{h}}_{1}+\mathbf{e}
$$

where $\tilde{\mathbf{h}}_{1}$ represents the actual target channel direction, whereas 416 $\hat{\mathbf{h}}_{1}$ is its quantized version, which is acquired from the user's 417 feedback. Vector e in (7) represents the quantization error, 418 which satisfies the ellipsoid constraint $\|\mathbf{e}\| \leq \varepsilon$. The quanti- 419 zation error for interfering channels can be defined similarly. 420 The problem is then solved using convex optimization, and 421 this technique is assumed to be known in this paper. This 422 traditional way of designing robust BF vectors does not meet 423 the assumptions stipulated in this paper. Earlier, we assumed 424 that the norms of the channel directions are 1 both before and 425 after quantization. This imposes more complex constraints on 426 the description of the quantization error. Hence, we conceive a 427 new technique of designing robust $\mathrm{BF}$ vectors for the scenario 428 when the CDI and CQI are fed back separately.

We adopt the random-vector-quantization codebook concept 430 [23] and use the model of [24] to analyze the quantization error, 431 where the quantization codebook index of B bits is linked with 432 the quantization error by

$$
\left|\left\langle\hat{\mathbf{h}}_{1}, \tilde{\mathbf{h}}_{1}\right\rangle\right| \geq 1-2^{\frac{-B}{K-1}} .
$$

When $\hat{\mathbf{h}}_{1}$ and $\hat{\mathbf{g}}_{1}$ are given, the problem of designing the 434 robust $\mathrm{BF}$ vector can be broken down into two separate parts. $\quad 435$

1) For each BF vector $\mathbf{w}$, find the set of $\tilde{\mathbf{h}}_{1}$ and $\tilde{\mathbf{g}}_{1}$ minimiz- 436 ing the SINR (denoted by $\operatorname{SINR}_{1}$ ) for this $\mathbf{w}$.

2) Find the $B F$ vector $w$, which ensures that this minimized 438 value of $\mathrm{SINR}_{1}$ is maximized.

The whole idea can be formulated as

$$
\mathbf{w}_{\text {opt }}=\arg \max _{\mathbf{w}}\left\{\min _{\tilde{\mathbf{h}}_{1}, \tilde{\mathbf{g}}_{1}}\left\{\operatorname{SINR}_{1}\left(\tilde{\mathbf{h}}_{1}, \tilde{\mathbf{g}}_{1} \mid \hat{\mathbf{h}}_{1}, \hat{\mathbf{g}}_{1}, \mathbf{w}\right)\right\}\right\} .
$$

Again, an intuitive illustration is given in Fig. 4. Since $\tilde{\mathbf{h}}_{1} 441$ and $\tilde{\mathbf{g}}_{1}$ are independent of each other, the problem of finding the 442 specific $\tilde{\mathbf{h}}_{1}$ and $\tilde{\mathbf{g}}_{1}$ that minimize the value of SINR for a given 443 


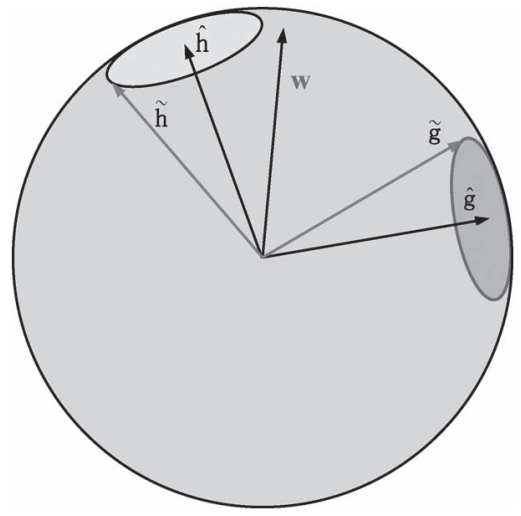

Fig. 4. Robust BF.

$444 \mathbf{w}$ is further simplified to finding $\tilde{\mathbf{h}}_{1}$ that minimizes $\left|\left\langle\mathbf{w}, \tilde{\mathbf{h}}_{1}\right\rangle\right|^{2}$ 445 and finding $\tilde{\mathbf{g}}_{1}$ that maximizes $\left|\left\langle\mathbf{w}, \tilde{\mathbf{g}}_{1}\right\rangle\right|^{2}$. The following lem446 mas provide a way of finding these $\tilde{\mathbf{h}}_{1}$ and $\tilde{\mathbf{g}}_{1}$ vectors.

447 Lemma 1: Upon assuming that $\left|\left\langle\mathbf{w}, \hat{\mathbf{g}}_{1}\right\rangle\right|=\lambda_{1}$ and that the 448 quantization error satisfies $\left|\left\langle\tilde{\mathbf{g}}_{1}, \hat{\mathbf{g}}_{1}\right\rangle\right|=\lambda_{2} \geq \lambda_{2 \text { min }} \geq \lambda_{1}$, we 449 have

$$
\left|\left\langle\mathbf{w}, \tilde{\mathbf{g}}_{1}\right\rangle\right| \leq \lambda_{1} \lambda_{2 \min }+\sqrt{\left(1-\lambda_{1}^{2}\right)\left(1-\lambda_{2 \min }^{2}\right)} .
$$

450 Proof: See Appendix B.

451 Lemma 2: Assuming that $\left|\left\langle\mathbf{w}, \hat{\mathbf{h}}_{1}\right\rangle\right|=\lambda_{1}$ and that the quan452 tization error satisfies $\left|\left\langle\tilde{\mathbf{h}}_{1}, \hat{\mathbf{h}}_{1}\right\rangle\right|=\lambda_{2} \geq \lambda_{2 \min } \geq \sqrt{1-\lambda_{1}^{2}}$, 453 we have

$$
\left|\left\langle\mathbf{w}, \tilde{\mathbf{h}}_{1}\right\rangle\right| \geq \lambda_{1} \lambda_{2 \min }-\sqrt{\left(1-\lambda_{1}^{2}\right)\left(1-\lambda_{2 \min }^{2}\right)} .
$$

454 Proof: See Appendix C.

455 In the given lemmas, $\lambda_{2}$ min $=1-2^{(-B / K-1)}$ represents the 456 maximum quantization error. With the aid of the given lemmas, 457 we have the following theorem.

458 Theorem 2: Upon introducing the notation of

$$
\mathbf{h}_{1}^{\dagger}=\frac{\hat{\mathbf{h}}_{1}-\left\langle\hat{\mathbf{h}}_{1}, \hat{\mathbf{g}}_{1}\right\rangle \hat{\mathbf{g}}_{1}}{\left\|\hat{\mathbf{h}}_{1}-\left\langle\hat{\mathbf{h}}_{1}, \hat{\mathbf{g}}_{1}\right\rangle \hat{\mathbf{g}}_{1}\right\|}
$$

459 and $\hat{\mathbf{h}}_{1}=\delta_{1} \mathbf{h}_{1}^{\dagger}+\delta_{2} \hat{\mathbf{g}}_{1}$, the optimal BF vector can be expressed 460 as a linear combination of vectors $\hat{\mathbf{h}}_{1}$ and $\hat{\mathbf{g}}_{1}$, which is formu461 lated as

$$
\mathbf{w}_{\mathrm{opt}}=\xi_{1} \mathbf{h}_{1}^{\dagger}+\xi_{2} \hat{\mathbf{g}}_{1}
$$

462 where $\xi_{1}$ and $\xi_{2}$ are appropriately chosen so that their norms 463 maximize the SINR in (14), shown at the bottom of the page, 464 whereas the arguments of $\xi_{1}$ and $\xi_{2}$ satisfy the constraint $465 \arg \left(\delta_{1}^{*} \xi_{1}\right)=\arg \left(\delta_{2}^{*} \xi_{2}\right)$.
Proof: See Appendix D.

The given theorem provides a numerical technique of design- 467 ing the optimal robust BF vector by finding the optimal value 468 of $\left|\xi_{2}\right|$, which falls in the real-valued interval of $[0,1]$.

\section{Extending the Algorithm to Multiple-BS Scenarios}

Earlier, we developed a low-complexity cross-layer algo- 471 rithm based on a scenario considering only two BSs. Let us 472 now show that this algorithm can be readily extended to more 473 general scenarios with multiple BSs involved.

Let us assume that there are $M$ BSs. In this multi-BS 475 extension of the algorithm, we still assume that there is a 476 single MBS, which cooperates with multiple SBSs, and that 477 all the BSs use the same BF vector. Additionally, since it 478 is reasonable to assume that different BSs have to transmit 479 independent messages to their scheduled users, we can treat the 480 interference at the $j$ th user of cell $k$ received from multiple BSs 481 as interference arriving from a single source associated with a 482 channel vector of

$$
\sum_{i=1, i \neq k}^{M} \mathbf{g}_{i, k, j} u_{i}
$$

where $\mathbf{g}_{i, k, j}$ is the channel vector of the link spanning from the 484 $i$ th BS to the $j$ th user in cell $k$, and $u_{i}$ is the symbol transmitted 485 to the scheduled user in cell $i$. Since the linear combination of 486 isotropic random vectors is also isotropic, the threshold-based 487 scheduling of Section III-A remains unchanged.

\section{Algorithmic Complexity AND SignALING OVERHEAD}

Here, we characterize the complexity of four algorithms: the 491 exhaustive search algorithm, the TDMA/FDMA scheme, the 492 iterative algorithm of [16], and our proposed algorithm.

493

Consider the scenario associated with $M$ BSs. The search for 494 the BF vector of a single BS is based on a searching through an 495 $L$-entry codebook, as implied in (14). Therefore, the exhaustive 496 search algorithm has complexity of $\mathcal{O}\left(L^{M} \prod_{i=1}^{M}\left|\mathcal{U}_{i}\right|\right)$, where 497 $\left|\mathcal{U}_{i}\right|$ is the supported number of users in the $i$ th BS, because 498 the algorithm will consider all possible user combinations to 499 find the one that achieves the optimal performance, which is 500 estimated based on an exhaustive search through the codebook 501 of each BS for an optimal combination of BF vectors for each 502 possible user combination.

503

The complexity of the iterative algorithm proposed in [16] 504 cannot be readily determined without knowing the number 505 of iterations needed for the convergence of the scheduling 506 process. Here, we introduce parameter $S$, which represents 507

$$
\operatorname{SINR}_{1}=\frac{\left.p_{11}\left\|\mathbf{h}_{1}\right\|^{2}\left(\lambda_{2 \min }\left(\left|\delta_{1} \xi_{1}\right|+\left|\delta_{2} \xi_{2}\right|\right)-\sqrt{\left(1-\lambda_{2 \min }^{2}\right)\left(1-\left(\left|\delta_{1} \xi_{1}\right|+\left|\delta_{2} \xi_{2}\right|\right)^{2}\right.}\right)\right)^{2}}{1+p_{21}\left\|\mathbf{g}_{1}\right\|^{2}\left(\left|\lambda_{2 \min } \xi_{2}\right|+\sqrt{\left(1-\lambda_{2 \min }^{2}\right)\left(1-\left|\xi_{2}\right|^{2}\right)}\right)^{2}}
$$


TABLE I

ALGORITHMIC COMPLEXITY

\begin{tabular}{|c|c|c|c|c|}
\hline Algorithm & Exhaustive Search & Iterative & TDMA/FDMA & Proposed \\
\hline Time(s) & $1.34 \mathrm{e} 3$ & $1.50 \mathrm{e} 1$ & $2.15 \mathrm{e}-1$ & $6.04 \mathrm{e}-1$ \\
\hline Complexity & $\mathcal{O}\left(L^{M} \prod_{i=1}^{M}\left|\mathcal{U}_{i}\right|\right)$ & $\mathcal{O}\left(S\left(L^{M}+\prod_{i=1}^{M}\left|\mathcal{U}_{i}\right|\right)\right)$ & $\mathcal{O}\left(\sum_{i=1}^{M}\left|\mathcal{U}_{i}\right|\right)$ & $\mathcal{O}\left(\left|\mathcal{U}_{1}\right| L+\sum_{i=2}^{M}\left|\mathcal{U}_{i}\right|\right)$ \\
\hline
\end{tabular}

TABLE II

SIGNALING OVERHEAD

\begin{tabular}{|c|c|c|c|c|c|c|}
\hline \multirow{2}{*}{\multicolumn{2}{|c|}{ Algorithm }} & \multirow{2}{*}{ Exhaustive search } & \multirow{2}{*}{ Iterative } & \multirow{2}{*}{ TDMA/FDMA } & \multicolumn{2}{|c|}{ Proposed } \\
\hline & & & & & BS1 (MBS) & $\mathrm{BS} i$ (SBS) \\
\hline \multicolumn{2}{|c|}{ CDI feedback per user } & $2 M$ & $2 M$ & 0 & 2 & 0 \\
\hline \multicolumn{2}{|c|}{ CQI feedback per user } & $2 M$ & $2 M$ & 1 & 2 & 1 \\
\hline \multicolumn{2}{|c|}{ Number of feedback users } & $\left|\mathcal{U}_{i}\right|$ & $\left|\mathcal{U}_{i}\right|$ & $\left|\mathcal{U}_{i}\right|$ & Greatly reduced & $\left|\mathcal{U}_{i}\right|$ \\
\hline \multirow{4}{*}{ Backhaul traffic } & CDI exchange & $\sum^{M} M\left|\mathcal{U}_{i}\right|$ & $M^{2}$ & 0 & \multicolumn{2}{|l|}{ 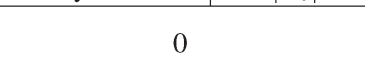 } \\
\hline & CQI exchange & $\sum_{i=1}^{M} M\left|\mathcal{U}_{i}\right|$ & $M^{2}$ & 0 & \multicolumn{2}{|c|}{0} \\
\hline & BF vector exchange & $M$ & $M$ & 0 & \multicolumn{2}{|c|}{$M-1$} \\
\hline & User identity exchange & $M$ & $M$ & 0 & \multicolumn{2}{|c|}{0} \\
\hline
\end{tabular}

508 the maximum affordable number of iterations for the algo509 rithm. Then, the resultant complexity may be expressed as $510 \mathcal{O}\left(S\left(L^{M}+\prod_{i=1}^{M}\left|\mathcal{U}_{i}\right|\right)\right)$, which follows from the fact that, each 511 time the BF vectors are updated, an exhaustive search of the 512 codebooks is conducted, whereas an exhaustive search for 513 the optimal user combination is carried out every time, when 514 the combination is updated.

515 The complexity of the TDMA/FDMA algorithms is $516 \mathcal{O}\left(\sum_{i=1}^{M}\left|\mathcal{U}_{i}\right|\right)$, which follows from the fact that each BS oper517 ates on its own and only has to set the BF vector to the direction 518 of the target channel of the scheduled user.

519 For the proposed algorithm, if we assume that BS1 is 520 the MBS, the upper bound of the complexity is $\mathcal{O}\left(\left|\mathcal{U}_{1}\right| L+\right.$ $\left.521 \sum_{i=2}^{M}\left|\mathcal{U}_{i}\right|\right)$. This is because the MBS only has a portion of 522 its users performing feedback, and the SBSs only have to find 523 the best users on their own by comparing their CQI feedback 524 values.

525 In the following, we characterize the algorithmic complexity 526 by monitoring the simulation time required for a single trial. We 527 conducted 1000 trials on all the algorithms aforementioned, and 528 in Table I, we listed the time required for each single trial for 529 CDI codebook sizes spanning from 2 to 15 . As we can see, the 530 exhaustive search algorithm and the iterative algorithm require 531 significantly more time than the noncooperative algorithm and 532 the proposed algorithm.

533 Finally, we compare the signaling overhead of the four given 534 algorithms in Table II, which includes the overhead generated 535 from users' feedback and the backhaul traffic. It is observed in 536 Table II that the exhaustive search algorithm and the iterative 537 algorithm require the same amount of feedback overhead, ex538 cept that the iterative algorithm generates less backhaul traffic 539 when the number of iterations is small. The overhead generated, 540 particularly the backhaul traffic, is enormous compared with the 541 noncooperative TDMA/FDMA schemes.

542 The proposed algorithm, on the other hand, effectively con543 trols the overhead generated from the CSI feedback and back544 haul traffic. Since the BF vectors are set to the same direction, 545 each user can view its received signals as if they were sent from only two sources, i.e., the destination and a single interfering 546 source. As a benefit, each user only has to feed back two sets of 547 channel information. Furthermore, the number of users generat- 548 ing feedback for the MBS is substantially reduced. A theoretical 549 expression for this number is hard to derive. Nonetheless, we 550 can determine with the aid of numerical simulations that, when 551 there are 20 users in each cell and the successful transmission 552 ratio is set to $99.9 \%$, the number of feedback users in the MBS 553 is, on average, 5.7. Meanwhile, the proposed algorithm only 554 requires the sharing of the BF vector selected by the MBS, 555 which is almost negligible compared with the backhaul traffic 556 generated by the iterative algorithm.

\section{Numerical Results}

Here, we first characterize the properties of threshold $T_{1}$, and 559 then quantify the performance of our proposed algorithm. $\quad 560$

\section{A. Threshold Value}

According to (5) and (6), the "Tropic" $T_{1}$ controls the trade- 562 off between the feedback load, algorithmic complexity, and the 563 probability of a successful DL scheduling action. From (6), $T_{1} 564$ is also a function of the number of transmit antennas and the 565 number of active users within the cell.

Fig. 5 shows the relation between the number of users and 567 the value of $T_{1}$, where the number of transmit antennas was set 568 to 2. Parameters $p_{i i}$ and $p_{j i}$ of both the MBS and the SBS are 569 set to $30 \mathrm{~dB}$, whereas the probability of success ranged from 570 $10 \%$ to $99.9 \%$. We can see in Fig. 5 that, when the probability 571 of success decreases, the threshold becomes stricter, and when 572 the number of users increases, the value of $T_{1}$ approaches 0.573 This is a manifestation of multiuser diversity since we are more 574 likely to have a user with better channel conditions when the 575 number of users becomes larger.

Fig. 6 shows the relationship between the probability of 577 successful DL scheduling and the percentage of reduction in 578 the CDI feedback per user. The numbers of users in both cells 579 range from 10 to 40 , whereas the number of transmit antennas 580 


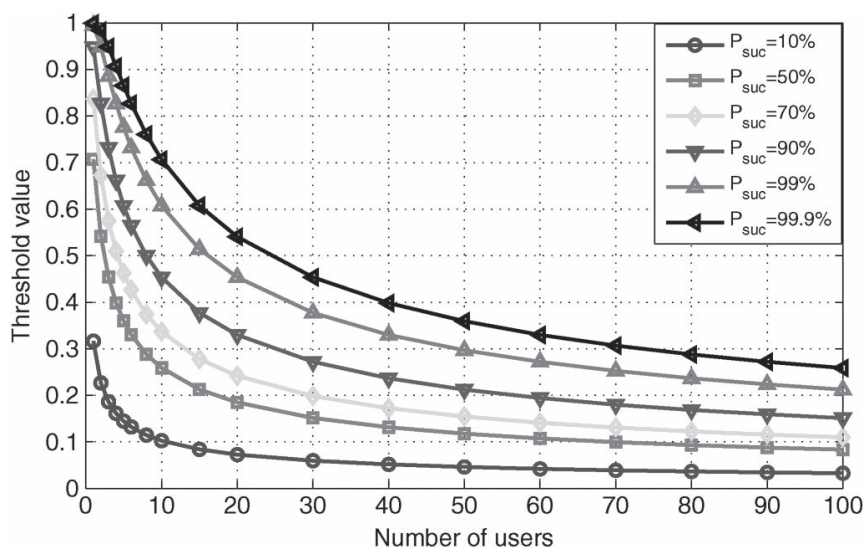

Fig. 5. Threshold value.

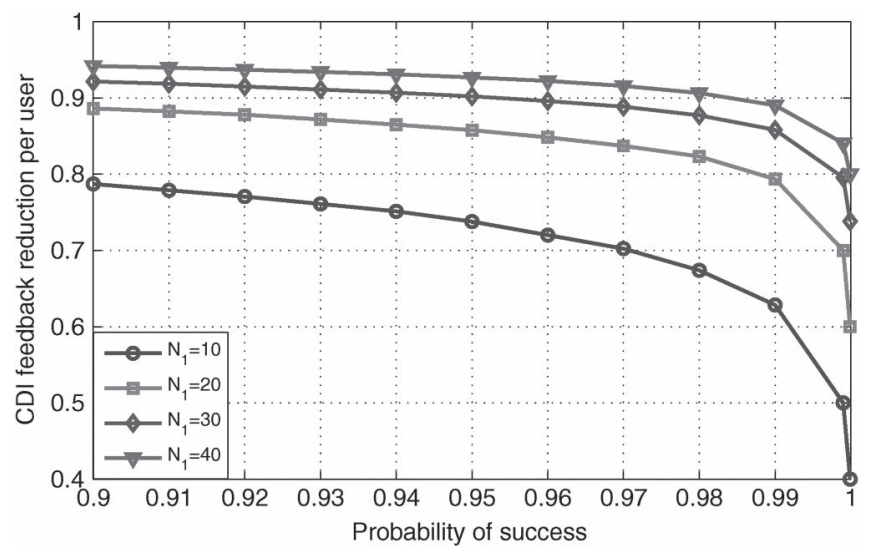

Fig. 6. Feedback load reduction per user.

581 remains 2, and the values $p_{i i}$ and $p_{j i}$ of both the MBS and the 582 SBS remain $30 \mathrm{~dB}$. Note that, in traditional TDMA and FDMA 583 schemes, all the users feed back their CSI; hence, the feedback 584 load per user is one CDI, where the number of bits is determined 585 by the codebook size. In CS/CB relying on exhaustive search, 586 the feedback load per user is two CDI times the number of 587 codebook index bits. In our proposed algorithm, however, the 588 feedback load per user is only $0.3 \mathrm{CDI}$, when the number of 589 users is 20 in both cells and the probability of success is $99.9 \%$. 590 This implies that, compared with TDMA and FDMA schemes, 591 the threshold $T_{1}$ allows us to rule out $70 \%$ of feedback without 592 undue degradation of the probability of success. Additionally, it 593 is possible to achieve an even lower feedback load by reducing 594 the probability of a successful DL scheduling, when the number 595 of users is given, but an excessive degradation of the probability 596 of success may ultimately impose throughput degradation.

\section{B. Throughput Performance}

598 Fig. 7 quantifies the throughput of the MBS relying on the 599 proposed algorithm in comparison to both the exhaustive search 600 scheme and the traditional TDMA and FDMA schemes. The 601 iterative algorithm proposed in [16] is used as a benchmark. 602 The number of users in both cells was set to 20, whereas 603 the parameters $p_{i i}$ and $p_{j i}$ of both the target $B S$ and of the 604 interfering $B S$ were set to $30 \mathrm{~dB}$, which was attenuated by 605 the path loss. The number of transmit antennas was two, and

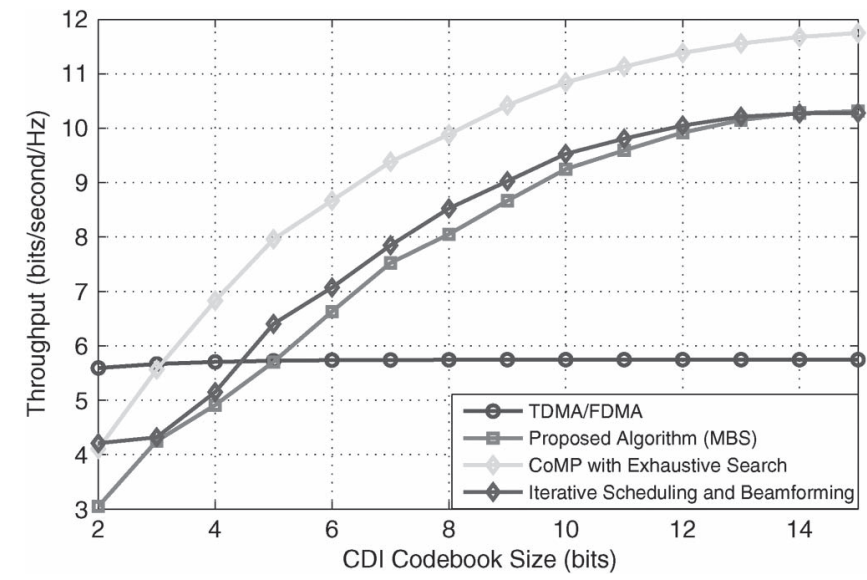

Fig. 7. DL throughput performance of the MBS.

the probability of DL scheduling success was set to $99.9 \% .606$ As shown in Fig. 7, the performance of the MBS does not 607 substantially degrade, and it is similar to the performance of the 608 iterative algorithm proposed in [16]. The discrepancy between 609 the exhaustive search algorithm and our proposed algorithm is a 610 result of striking a tradeoff between the algorithmic complexity, 611 overhead, and performance. Again, the discrepancy between 612 the iterative algorithm and the exhaustive search algorithm is 613 contributed by both the imperfect CSI feedback and by the 614 fact that the iterative algorithm cannot guarantee consistent 615 convergence to the global optimum. We also note that the left- 616 hand part of the curves is not as smooth as their right-hand 617 part. When the size of the codebook is 2 bits, the iterative 618 algorithm even yields a slightly better performance than the 619 exhaustive search algorithm. This, however, does not mean 620 that the iterative algorithm performs in general better than the 621 exhaustive search algorithm. This phenomenon is due to the 622 large quantization errors of the CDI feedback. While the ex- 623 haustive search algorithm did guarantee the maximization of the 624 minimum throughput of the scheduled users, the maximization 625 of the actual performance is evaluated with the aid of the actual 626 channel conditions encountered by the scheduled users. Hence, 627 we conclude that the better performance of the iterative algo- 628 rithm is a result of the large quantization errors imposed by the 629 limited codebook size of the CDI feedback. This phenomenon 630 does not occur when the codebook size is large.

631

Furthermore, observe in Fig. 7 that when the CDI codebook 632 size is small, the performance of cooperative $\mathrm{BF}$ is similar to or 633 even lower than that of the noncooperative TDMA and FDMA 634 schemes. This phenomenon raises the question as to what is the 635 minimum amount of feedback required by cooperative trans- 636 mission and scheduling schemes to outperform their traditional 637 noncooperative counterparts. This question is studied in detail 638 in [15].

Fig. 8 shows the performance comparison of an SBS and 640 the performance of a regular BS running under TDMA/FDMA 641 schemes. The codebook size is set to 15 in this case, whereas 642 the performances with other codebook sizes are similar. It can 643 be easily seen that the performance of the SBS is sometimes 644 not as good as the regular BS but becomes better as the number 645 of users increases. This is caused by the multiuser diversity 646 effect. It can be also observed that the intersection of the 647 


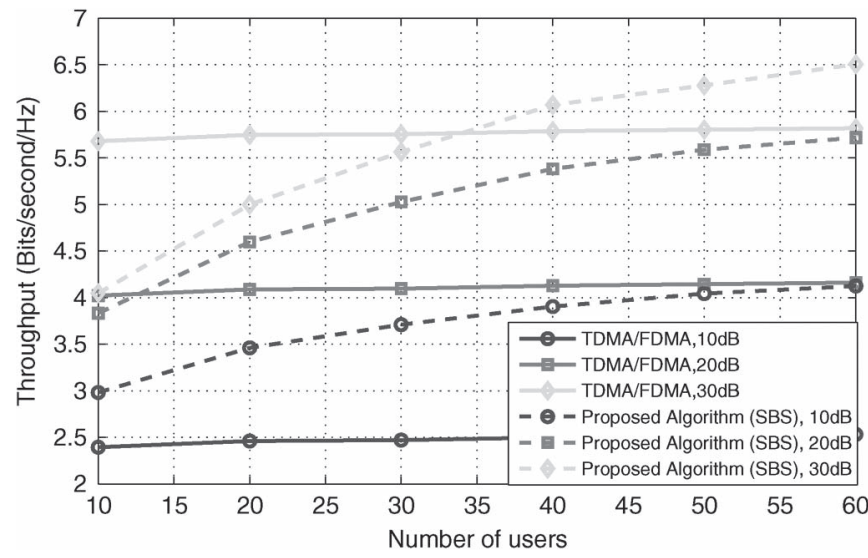

Fig. 8. Performance comparison between an SBS and a BS running under TDMA/FDMA schemes.

648 curves of the performances of the SBS and the regular BS 649 shifts toward right as the SNR increases, which means that at 650 high SNR levels, the proposed scheme will need more users 651 to beat the performance of the TDMA/FDMA schemes. This 652 phenomenon can be interpreted by the suboptimality of the $653 \mathrm{BF}$ vector. For a small amount of the given users, the effect 654 of $\mathrm{BF}$ is dominating when SNR level is low. However, as 655 SNR increases, the suboptimality of the direction of the BF 656 vector becomes a major constraint to improve the performance, 657 and the proposed algorithm is surpassed by the TDMA/FDMA 658 schemes gradually.

\section{Conclusion}

660 In this paper, we have conceived a low-complexity cross661 layer algorithm for DL CoMP, which promises a good per662 formance for the MBS while significantly reducing both the 663 amount of feedback and the algorithmic complexity. The 664 scheduling scheme of the proposed algorithm efficiently ex665 ploited the knowledge of CSI at the receiver. For the BF part of 666 the algorithm, we provided a new technique of designing robust $667 \mathrm{BF}$ vectors, when the CDI and CQI of the users are fed back 668 to the BSs separately. Our numerical results demonstrated that 669 our solution only moderately degraded the performance of the 670 potentially excessive-complexity exhaustive search technique, 671 despite having complexity as low as that of a conventional 672 system operating without CoMP. We next present the proofs 673 of the theorems stated earlier.

674

675

676 For the MBS, denote the channel directions of the sched677 uled user as $\tilde{\mathbf{h}}_{1}=\left[h_{1}, h_{2}, \ldots, h_{K}\right]^{T}, \tilde{\mathbf{g}}_{1}=\left[g_{1}, g_{2}, \ldots, g_{K}\right]^{T}$. 678 Since $\mathbf{h}_{1} \sim C N\left(0, \mathbf{I}_{K}\right)$, it follows that $\left[\operatorname{Re}\left(h_{i}\right), \operatorname{Im}\left(h_{i}\right)\right]^{T} \sim$ $679 N\left(0,\left(1 / 2 \mathbf{I}_{2}\right)\right.$ and $\left[\operatorname{Re}\left(g_{i}\right), \operatorname{Im}\left(g_{i}\right)\right]^{T} \sim N\left(0,(1 / 2) \mathbf{I}_{2}\right)$. Thus, 680 the random vector $\tilde{\mathbf{h}}_{1}$ spanning the complex space $\mathbb{C}^{K}$ equals

${ }^{1}$ We discovered that similar results are derived in [25], where the authors directly computed the surface area of the unit hypersphere and spherical cap in complex space. The absolute surface area is not the same when considered in a $K$-D complex space and a $2 K$-D real space, but the resulting probability is the same. a random vector confined to the real space, which can be 681 formulated as $\overline{\mathbf{h}}_{1} \in \mathbb{R}^{2 K}$. The real random vector $\overline{\mathbf{h}}_{1}$ obeys the 682 normal distribution of $N\left(0,(1 / 2) \mathbf{I}_{2 K}\right)$.

According to Section III-A, the goal of the scheduling al- 684 gorithm is to find the specific user, whose channel directions 685 $\widetilde{\mathbf{h}}_{1}$ and $\tilde{\mathbf{g}}_{1}$ are "most different" from each other. Assuming 686 $\left|\left\langle\tilde{\mathbf{h}}_{1}, \tilde{\mathbf{g}}_{1}\right\rangle\right|=|\cos \theta|$, the probability density function of $|\cos \theta| 687$ can be expressed as

$$
f(|\cos \theta|)=f\left(\left|\sum_{i=1}^{K} h_{i} g_{i}^{*}\right|\right)=f\left(\sqrt{A_{3}^{2}+A_{4}^{2}}\right)
$$

where $f(\cdot)$ denotes the probability density function of any 689 random variable or random vector, and

$$
\begin{aligned}
& A_{3}=\sum_{i=1}^{K}\left[\operatorname{Re}\left(h_{i}\right) \operatorname{Re}\left(g_{i}\right)+\operatorname{Im}\left(h_{i}\right) \operatorname{Im}\left(g_{i}\right)\right] \\
& A_{4}=\sum_{i=1}^{K}\left[\operatorname{Re}\left(h_{i}\right) \operatorname{Im}\left(g_{i}\right)-\operatorname{Re}\left(g_{i}\right) \operatorname{Im}\left(h_{i}\right)\right] .
\end{aligned}
$$

Now, if we define three new random vectors in the set of $\mathbb{R}^{2 K} 691$

$$
\begin{aligned}
\overline{\mathbf{h}}^{(1)} & =\left[\operatorname{Re}\left(h_{1}\right), \ldots, \operatorname{Re}\left(h_{K}\right), \operatorname{Im}\left(h_{1}\right), \ldots, \operatorname{Im}\left(h_{K}\right)\right]^{T} \\
\overline{\mathbf{g}}^{(1)} & =\left[\operatorname{Re}\left(g_{1}\right), \ldots, \operatorname{Re}\left(g_{K}\right), \operatorname{Im}\left(g_{1}\right), \ldots, \operatorname{Im}\left(g_{K}\right)\right]^{T} \\
\overline{\mathbf{g}}^{(2)} & =\left[\operatorname{Im}\left(g_{1}\right), \ldots, \operatorname{Im}\left(g_{K}\right),-\operatorname{Re}\left(g_{1}\right), \ldots,-\operatorname{Re}\left(g_{K}\right)\right]
\end{aligned}
$$

then, (16) can be further simplified as

$$
f(|\cos \theta|)=f\left(\sqrt{\left|\left(\overline{\mathbf{h}}^{(1)}\right)^{T} \overline{\mathbf{g}}^{(1)}\right|^{2}+\left|\left(\overline{\mathbf{h}}^{(1)}\right)^{T} \overline{\mathbf{g}}^{(2)}\right|^{2}}\right) .
$$

Since we have $\left|\left(\overline{\mathbf{g}}^{(1)}\right)^{T} \overline{\mathbf{g}}^{(2)}\right|=0$, there exists $(2 K-693$ 2) real-valued vectors with unit norms of $\overline{\mathbf{g}}^{(3)}, \ldots, \overline{\mathbf{g}}^{(2 K)}, 694$ which are orthogonal to each other, including $\overline{\mathbf{g}}^{(1)}$ and 695 $\overline{\mathbf{g}}^{(2)}$. Thus, by letting $\mathbf{M}=\left[\overline{\mathbf{g}}^{(1)}, \overline{\mathbf{g}}^{(2)}, \ldots, \overline{\mathbf{g}}^{(2 K)}\right]$, we have 696 $\left|\left(\overline{\mathbf{h}}^{(1)}\right)^{T} \mathbf{M} \mathbf{M}^{T} \overline{\mathbf{g}}^{(1)}\right|^{2}=\operatorname{Re}\left(h_{1}\right)^{2}$ and $\left|\left(\overline{\mathbf{h}}^{(1)}\right)^{T} \mathbf{M M}^{T} \overline{\mathbf{g}}^{(2)}\right|^{2}=697$ $\operatorname{Im}\left(h_{1}\right)^{2}$; thus

$f(|\cos \theta|)=f\left(\sqrt{\operatorname{Re}\left(h_{1}\right)^{2}+\operatorname{Im}\left(h_{1}\right)^{2}} \mid \overline{\mathbf{g}}^{(1)}\right) f\left(\overline{\mathbf{g}}^{(1)}\right)$.

This means that the value of $|\cos \theta|^{2}$ equals the sum of the 699 squares of the two coordinates of $\overline{\mathbf{h}}^{(1)}$ along the two orthog- 700 onal dimensions. Additionally, note that the direction of the 701 random vector $\overline{\mathbf{h}}^{(1)}$ is isotropic [26], which implies that the 702 probability density function of $\tilde{\mathbf{h}}_{1}$ on the surface of the $2 K$-D 703 hypersphere with unit radius is $1 / S_{2 K}$, where we have 704 $S_{2 K}=2 K \pi^{K} / \Gamma(1+K)$. If we define $S_{\operatorname{Re}\left(h_{1}\right)^{2}+\operatorname{Im}\left(h_{1}\right)^{2} \leq T_{1}^{2} 705}$ and $S_{\operatorname{Re}\left(h_{1}\right)^{2}+\operatorname{Im}\left(h_{1}\right)^{2}=r^{2}}$ to be the surface area of the hyper- 706 sphere satisfying the constraint described in the subscript, the 707 probability that a specific user's channel directions satisfy the 708 threshold constraint denoted by $P_{1}$ can be expressed as

$$
\begin{aligned}
P_{1} & =P\left(\sqrt{\operatorname{Re}\left(\mathrm{h}_{1}\right)^{2}+\operatorname{Im}\left(\mathrm{h}_{1}\right)^{2}} \leq T_{1}\right) \\
& =\frac{S_{\operatorname{Re}\left(h_{1}\right)^{2}+\operatorname{Im}\left(h_{1}\right)^{2} \leq T_{1}^{2}}}{S_{2 K}} .
\end{aligned}
$$


710 By exploiting

$$
\begin{aligned}
& S_{\operatorname{Re}\left(h_{1}\right)^{2}+\operatorname{Im}\left(h_{1}\right)^{2} \leq T_{1}^{2}} \\
& =\int_{0}^{T_{1}} S_{\operatorname{Re}\left(h_{1}\right)^{2}+\operatorname{Im}\left(h_{1}\right)^{2}=r^{2} d r} \\
& =\int_{0}^{\arcsin T_{1}}\left[\frac{2(K-1) \pi^{K-1} r^{2 K-3}}{\Gamma(K)}\right]_{r=\cos \theta} 2 \pi \sin \theta d \theta \\
& =\frac{2 \pi^{K}\left(1-\left(1-T_{1}^{2}\right)^{K-1}\right)}{\Gamma(K)}
\end{aligned}
$$

711 we arrive at

$$
P_{1}=1-\left(1-T_{1}^{2}\right)^{K-1}
$$

712 Since there are $N_{1}$ users in the cell, the probability that there 713 is at least one user that satisfies the threshold constraint can be 714 expressed as

$$
P_{\text {suc1 }}=1-\left(1-P_{1}\right)^{N_{1}} \text {. }
$$

715 With the aid of (23), we finally have

$$
P_{\text {suc } 1}=1-\left(1-T_{1}^{2}\right)^{N_{1}(K-1)} .
$$

\section{6 \\ 717 \\ APPENDIX B \\ PROOF OF LEMMA 1}

718 Let

$$
\mathbf{w}_{1}=\frac{\mathbf{w}-\left\langle\mathbf{w}, \hat{\mathbf{g}}_{1}\right\rangle \hat{\mathbf{g}}_{1}}{\left\|\mathbf{w}-\left\langle\mathbf{w}, \hat{\mathbf{g}}_{1}\right\rangle \hat{\mathbf{g}}_{1}\right\|}
$$

719 Then, $\mathbf{w}_{1}$ is orthogonal to $\hat{\mathbf{g}}_{1}$, and it can be expressed as a linear 720 combination of $\hat{\mathbf{g}}_{1}$ and $\mathbf{w}$. Thus, we can assume that

$$
\begin{aligned}
& \mathbf{w}=\varphi_{1} \mathbf{w}_{1}+\varphi_{2} \hat{\mathbf{g}}_{1} \\
& \tilde{\mathbf{g}}_{1}=\theta_{1} \mathbf{w}_{1}+\theta_{2} \hat{\mathbf{g}}_{1}+\theta_{3} \mathbf{u}
\end{aligned}
$$

721 where vector $\mathbf{u}$ is an arbitrary vector with unit norm and 722 orthogonal to both $\hat{\mathbf{g}}_{1}$ and $\mathbf{w}$. Additionally, we should also note 723 that, in the given equations

$$
\left|\varphi_{1}\right|^{2}+\left|\varphi_{2}\right|^{2}=\left|\theta_{1}\right|^{2}+\left|\theta_{2}\right|^{2}+\left|\theta_{3}\right|^{2}=1 \text {. }
$$

724 Thus, we have

$$
\left|\left\langle\mathbf{w}, \tilde{\mathbf{g}}_{1}\right\rangle\right|=\left|\varphi_{1} \theta_{1}^{*}+\varphi_{2} \theta_{2}^{*}\right| \leq\left|\varphi_{1} \theta_{1}\right|+\left|\varphi_{2} \theta_{2}\right|
$$

725 where the equality on the right side holds if and only if we have

$$
\arg \left(\varphi_{1} \theta_{1}^{*}\right)=\arg \left(\varphi_{2} \theta_{2}^{*}\right)
$$

726 According to (28), we have

$$
\left|\left\langle\tilde{\mathbf{g}}_{1}, \hat{\mathbf{g}}_{1}\right\rangle\right|=\left|\theta_{2}\right|=\lambda_{2} \geq \lambda_{2 \min } \geq \lambda_{1}=\left|\varphi_{2}\right| \text {. }
$$

We can see from the given equation that the maximum 727 value of $\left|\left\langle\mathbf{w}, \tilde{\mathbf{g}}_{1}\right\rangle\right|$ is achieved when $\theta_{3}$ is zero. If not, we 728 can force $\theta_{3}$ to zero and multiply both $\theta_{1}$ and $\theta_{2}$ by a factor 729 of $1 / \sqrt{1-\left|\theta_{3}\right|^{2}}$. According to (30), the value of $\left|\left\langle\mathbf{w}, \tilde{\mathbf{g}}_{1}\right\rangle\right| 730$ increases. Since $\left|\theta_{2}\right|$ is always larger than $\left|\varphi_{2}\right|$, according to 731 Chebyshev's inequality, we can see that increasing the value 732 of $\left|\varphi_{2}\right|$ will result in a monotonic increase in the value of 733 $\left|\left\langle\mathbf{w}, \tilde{\mathbf{g}}_{1}\right\rangle\right|$. Thus, the maximum value of $\left|\left\langle\mathbf{w}, \tilde{\mathbf{g}}_{1}\right\rangle\right|$ is achieved, 734 when $\lambda_{2}=\lambda_{2 \text { min. }}$. This means that the maximum value of 735 $\left|\left\langle\mathbf{w}, \tilde{\mathbf{g}}_{1}\right\rangle\right|$ is achieved, when the actual channel direction falls 736 on the quantization cell boundary between the BF vector and 737 the unit vector representing the quantized channel direction. 738

\section{APPENDIX C \\ PROOF OF LEMMA 2}

Lemma 2 can be proven in the same way as Lemma 1. Let 741

$$
\mathbf{w}_{1}=\frac{\mathbf{w}-\left\langle\mathbf{w}, \hat{\mathbf{h}}_{1}\right\rangle \hat{\mathbf{h}}_{1}}{\left\|\mathbf{w}-\left\langle\mathbf{w}, \hat{\mathbf{h}}_{1}\right\rangle \hat{\mathbf{h}}_{1}\right\|} .
$$

Then, vector $\mathbf{w}_{1}$ is orthogonal to $\hat{\mathbf{h}}_{1}$, and it can be expressed as 742 a linear combination of $\mathbf{w}$ and $\hat{\mathbf{h}}_{1}$. Thus, we can assume that 743

$$
\begin{aligned}
\mathbf{w} & =\varphi_{3} \mathbf{w}_{1}+\varphi_{4} \hat{\mathbf{h}}_{1} \\
\tilde{\mathbf{h}}_{1} & =\theta_{4} \mathbf{w}_{1}+\theta_{5} \hat{\mathbf{h}}_{1}+\theta_{6} \mathbf{u}
\end{aligned}
$$

where vector $\mathbf{u}$ is an arbitrary vector with a unit norm and 744 orthogonal to both $\hat{\mathbf{h}}_{1}$ and $\mathbf{w}$. Since the norms of both $\mathbf{w}$ and 745 $\tilde{\mathbf{h}}_{1}$ are 1 , it easily follows that

$$
\left|\varphi_{3}\right|^{2}+\left|\varphi_{4}\right|^{2}=\left|\theta_{4}\right|^{2}+\left|\theta_{5}\right|^{2}+\left|\theta_{6}\right|^{2}=1 \text {. }
$$

Thus, we have

$$
\left|\left\langle\mathbf{w}, \tilde{\mathbf{h}}_{1}\right\rangle\right|=\left|\varphi_{3} \theta_{4}^{*}+\varphi_{4} \theta_{5}^{*}\right| \geq\left\|\varphi_{3} \theta_{4}|-| \varphi_{4} \theta_{5}\right\| .
$$

It is clear from (34) and (35) that $\left|\varphi_{4}\right|=\left|\lambda_{1}\right|$ and $\left|\theta_{5}\right|=\lambda_{2} .748$ Since $\lambda_{2} \geq \sqrt{1-\lambda_{1}^{2}}$, we have $\lambda_{1} \geq \sqrt{1-\lambda_{2}^{2}}$. Thus

$$
|| \varphi_{3} \theta_{4}|-| \varphi_{4} \theta_{5} \|=\lambda_{1} \lambda_{2}-\sqrt{\left(1-\lambda_{1}^{2}\right)\left(1-\lambda_{2}^{2}-\left|\theta_{6}\right|^{2}\right)} .
$$

In the given equation, we first observe that when $\left|\left\langle\mathbf{w}, \tilde{\mathbf{h}}_{1}\right\rangle\right| 750$ is minimized, $\left|\theta_{6}\right|$ has to be zero. This is obvious since, 751 for a given $\lambda_{1}$, if we hold $\lambda_{2}$ at a fixed value smaller 752 than 1 , increasing the value of $\left|\theta_{6}\right|$ will result in a de-753 crease in $\sqrt{\left(1-\lambda_{1}^{2}\right)\left(1-\lambda_{2}^{2}-\left|\theta_{6}\right|^{2}\right)}$, and if the value of 754 $\left|\left\langle\mathbf{w}, \tilde{\mathbf{h}}_{1}\right\rangle\right|$ is minimized, $\left|\theta_{6}\right|$ increases. Additionally, when 755 $\left|\left\langle\mathbf{w}, \tilde{\mathbf{h}}_{1}\right\rangle\right|$ is minimized, $\left|\theta_{6}\right|$ is minimized, and $\lambda_{2}=\lambda_{2 \min } .756$ This is because when $\lambda_{2}$ decreases, $\lambda_{1} \lambda_{2}$ decreases, and 757 $\sqrt{\left(1-\lambda_{1}^{2}\right)\left(1-\lambda_{2}^{2}-\left|\theta_{6}\right|^{2}\right)}$ increases at the same time.

758

With the given observations, the lemma is proven. Note that 759 this lemma tells us that the minimum of $\left|\left\langle\mathbf{w}, \tilde{\mathbf{h}}_{1}\right\rangle\right|$ is achieved 760 when the actual channel direction $\tilde{\mathbf{h}}_{1}$ is away from both the BF 761 vector $\mathbf{w}$ and the quantized channel direction $\hat{\mathbf{h}}_{1}$ and falls on 762 the quantization cell boundary. 


$$
\operatorname{SINR}_{1}=\frac{p_{11}\left\|\mathbf{h}_{1}\right\|^{2}\left(\lambda_{2 \min }\left|\xi_{5}\right|-\sqrt{\left(1-\lambda_{2 \min }^{2}\right)\left(1-\left|\xi_{5}\right|^{2}\right)}\right)^{2}}{1+p_{21}\left\|\mathbf{g}_{1}\right\|^{2}\left(\left|\lambda_{2 \min } \xi_{2}\right|+\sqrt{\left(1-\lambda_{2 \min }^{2}\right)\left(1-\left|\xi_{2}\right|^{2}\right)}\right)^{2}}
$$

Let

$$
\mathbf{g}_{1}^{\dagger}=\frac{\hat{\mathbf{g}}_{1}-\left\langle\hat{\mathbf{g}}_{1}, \hat{\mathbf{h}}_{1}\right\rangle \hat{\mathbf{h}}_{1}}{\left\|\hat{\mathbf{g}}_{1}-\left\langle\hat{\mathbf{g}}_{1}, \hat{\mathbf{h}}_{1}\right\rangle \hat{\mathbf{h}}_{1}\right\|}
$$

767 where $\mathbf{h}_{1}^{\dagger}$ is orthogonal to $\hat{\mathbf{g}}_{1}$ and can be expressed as a linear 768 combination of $\hat{\mathbf{g}}_{1}$ and $\hat{\mathbf{h}}_{1}$. Vector $\mathbf{g}_{1}^{\dagger}$ is orthogonal to $\hat{\mathbf{h}}_{1}$ and 769 can be also expressed as a linear combination of $\hat{\mathbf{g}}_{1}$ and $\hat{\mathbf{h}}_{1}$. 770 Assuming that vector $\mathbf{u}_{1}$ is an arbitrary vector with a unit norm 771 and orthogonal to both $\mathbf{h}_{1}^{\dagger}$ and $\hat{\mathbf{h}}_{1}$ and that vector $\mathbf{u}_{2}$ is an 772 arbitrary vector with a unit norm and orthogonal to both $\mathbf{g}_{1}^{\dagger}$ and $773 \hat{\mathrm{g}}_{1}$, we have

$$
\begin{aligned}
\mathbf{w} & =\xi_{1} \mathbf{h}_{1}^{\dagger}+\xi_{2} \hat{\mathbf{g}}_{1}+\xi_{3} \mathbf{u}_{1}=\xi_{4} \mathbf{g}_{1}^{\dagger}+\xi_{5} \hat{\mathbf{h}}_{1}+\xi_{6} \mathbf{u}_{2} \\
\hat{\mathbf{h}}_{1} & =\delta_{1} \mathbf{h}_{1}^{\dagger}+\delta_{2} \hat{\mathbf{g}}_{1} \\
\hat{\mathbf{g}}_{1} & =\delta_{3} \mathbf{g}_{1}^{\dagger}+\delta_{4} \hat{\mathbf{h}}_{1} .
\end{aligned}
$$

774 In the given equations, since the norms of $\mathbf{w}, \hat{\mathbf{h}}_{1}$, and $\hat{\mathbf{g}}_{1}$ are all 7751 , it follows that

$$
\begin{aligned}
& \sum_{i=1}^{3}\left|\xi_{i}\right|^{2}=\sum_{i=4}^{6}\left|\xi_{i}\right|^{2}=1 \\
& \left|\delta_{1}\right|^{2}+\left|\delta_{2}\right|^{2}=\left|\delta_{3}\right|^{2}+\left|\delta_{4}\right|^{2}=1 .
\end{aligned}
$$

776 Thus, with the aid of (40) to (44) and the two lemmas, for a 777 given set of $\hat{\mathbf{g}}_{1}$ and $\hat{\mathbf{h}}_{1}$, the minimum of $\mathrm{SINR}_{1}$ takes the form 778 of (45), shown at the top of the page. Additionally, we also have

$$
\left|\xi_{5}\right|=\left|\left\langle\mathbf{w}, \hat{\mathbf{h}}_{1}\right\rangle\right|=\left|\xi_{1} \delta_{1}^{*}+\xi_{2} \delta_{2}^{*}\right| \leq\left|\xi_{1} \delta_{1}\right|+\left|\xi_{2} \delta_{2}\right| .
$$

779 When the minimum SINR in (45) is maximized, the equality 780 on the right side of (46) holds. This is because, when $\left|\xi_{5}\right|$ 781 increases, the numerator of (45) increases, and increasing the 782 value of $\left|\xi_{5}\right|$ can be achieved by changing only the principles of $783 \xi_{1}$ and $\xi_{2}$, which will not affect the value of the denominator in 784 the equation. Additionally, we observe that, when the minimum 785 SINR in (45) is maximized, we have $\left|\xi_{3}\right|=0$. The proof 786 exploits that, if the maximum value of this $\mathrm{SINR}_{1}$ is achieved 787 when the BF vector $\mathbf{w}$ is not on the same complex plane with 788 both $\hat{\mathbf{g}}_{1}$ and $\hat{\mathbf{h}}_{1}$, we have $\left|\xi_{3} \xi_{6}\right| \neq 0$. In this case, we can hold $789\left|\xi_{2}\right|$ at a fixed value, and set $\xi_{3}$ to 0 . This will result in an 790 increase in $\left|\xi_{1}\right|$, and since

$$
\left|\xi_{5}\right|=\left|\delta_{1} \xi_{1}\right|+\left|\delta_{2} \xi_{2}\right|
$$

791 the value of $\left|\xi_{5}\right|$ increases, resulting in an increase in the value 792 of SINR $_{1}$. Upon combining (45) and (47), we arrive at (14); 793 hence, the theorem is proven.

\section{REFERENCES}

[1] M. Sawahashi, Y. Kishiyama, A. Morimoto, D. Nishikawa, and 795 M. Tanno, "Coordinated multipoint transmission/reception techniques for 796 LTE-advanced," IEEE Wireless Commun., vol. 17, no. 3, pp. 26-34, 797 Jun. 2010.

798

[2] R. Irmer, H. Droste, P. Marsch, M. Grieger, G. Fettweis, S. Brueck, 799 H. P. Mayer, L. Thiele, and V. Jungnickel, "Coordinated multipoint: Con- 800 cepts, performance, and field trial results," IEEE Commun. Mag., vol. 49, 801 no. 2, pp. 102-111, Feb. 2011.

802

[3] D. Choi, D. Lee, and J. H. Lee, "Resource allocation for CoMP with 803 multiuser MIMO-OFDMA," IEEE Trans. Veh. Technol., vol. 60, no. 9, 804 pp. 4626-4632, Nov. 2011.

805

[4] R. Bhagavatula and R. W. Heath, "Adaptive limited feedback for sum-rate 806 maximizing beamforming in cooperative multicell systems," IEEE Trans. 807 Signal Process., vol. 59, no. 2, pp. 800-811, Feb. 2011.

[5] D. Love, R. Heath, V. Lau, D. Gesbert, B. Rao, and M. Andrews, "An 809 overview of limited feedback in wireless communication systems," IEEE 810 J. Sel. Areas Commun., vol. 26, no. 8, pp. 1341-1365, Oct. 2008.

811

[6] T. Wu and V. Lau, "Design and analysis of multi-user SDMA systems 812 with noisy limited CSIT feedback," IEEE Trans. Wireless Commun, vol. 9, 813 no. 4, pp. 1446-1450, Apr. 2010.

[7] T. Wu and V. Lau, "Robust precoder adaptation for MIMO links with 815 noisy limited feedback," IEEE Trans. Inf. Theory, vol. 55, no. 4, pp. 1640- 816 1649, Apr. 2009.

[8] C. Murthy, J. Zheng, and B. Rao, "Performance of quantized equal gain 818 transmission with noisy feedback channels," IEEE Trans. Signal Process., 819 vol. 56, no. 6, pp. 2451-2460, Jun. 2008.

820

[9] A. Housfater and T. J. Lim, "Throughput of precoded broadcast transmis- 821 sion with noisy feedback," in Proc. IEEE Int. Symp. Inf. Theory, Jul. 2009, 822 pp. 398-402.

[10] A. Housfater and T. J. Lim, "Noisy feedback linear precoding: A 824 bayesian cramér-rao bound," in Proc. Int. Symp. Inf. Theory, Jul. 2009, 825 pp. 1689-1693

[11] I. S. Dhillon, R. W. Heath, T. Strohmer, and J. A. Tropp, “Constructing 827 packings in Grassmannian manifolds via alternating projection," Exp. 828 Math., vol. 17, no. 7, pp. 9-35, 2008.

[12] Y. Cheng, V. Lau, and Y. Long, "A scalable limited feedback design for 830 network MIMO using per-cell product codebook," IEEE Trans. Wireless 831 Commun., vol. 9, no. 10, pp. 3093-3099, Oct. 2010.

[13] X. Hou and C. Yang, "Codebook design and selection for multi-cell 833 cooperative transmission limited feedback systems," in Proc. IEEE 73rd 834 VTC, May 2011, pp. 1-5.

[14] D. Su, X. Hou, and C. Yang, "Quantization based on per-cell codebook 836 in cooperative multi-cell systems," in Proc. IEEE WCNC, Mar. 2011, 837 pp. 1753-1758.

838

[15] X. Hou and C. Yang, "How much feedback overhead is required for base 839 station cooperative transmission to outperform non-cooperative transmis- 840 sion?" in Proc. IEEE ICASSP, May 2011, pp. 3416-3419.

[16] W. Yu, T. Kwon, and C. Shin, "Multicell coordination via joint scheduling, 842 beamforming and power spectrum adaptation," in Proc. IEEE INFOCOM, 843 Apr. 2011, pp. 2570-2578.

[17] P. Viswanath, D. Tse, and R. Laroia, "Opportunistic beamforming using 845 dumb antennas," IEEE Trans. Inf. Theory, vol. 48, no. 6, pp. 1277-1294, 846 Jun. 2002.

[18] D. Park and G. Caire, "Hard fairness versus proportional fairness in wire- 848 less communications: The multiple-cell case," in Proc. IEEE Int. Symp. 849 Inf. Theory, Jul. 2008, pp. 2036-2040.

[19] S. A. Vorobyov, A. B. Gershman, and Z.-Q. Luo, "Robust adaptive beam- 851 forming using worst-case performance optimization: a solution to the 852 signal mismatch problem," IEEE Trans. Signal Process., vol. 51, no. 2, 853 pp. 313-324, Feb. 2003.

[20] R. Lorenz and S. Boyd, "Robust minimum variance beamforming," IEEE 855 Trans. Signal Process, vol. 53, no. 5, pp. 1684-1696, May 2005.

[21] A. Abdel-Samad, A. B. Gershman, and T. N. Davidson, "Robust transmit 857 beamforming based on imperfect channel feedback," in Proc. IEEE 60th 858 VTC-Fall, Sep. 2004, vol. 3, pp. 2049-2053. 
860 [22] E. Gharavol, Y.-C. Liang, and K. Mouthaan, "Robust downlink beam861 forming in multiuser MISO cognitive radio networks with imperfect 862 channel-state information," IEEE Trans. Veh. Technol., vol. 59, no. 6, 863 pp. 2852-2860, Jul. 2010.

864 [23] W. Santipach and M. Honig, "Asymptotic capacity of beamforming with 865 limited feedback," in Proc. ISIT, Jul. 2004, p. 290.

866 [24] T. Yoo, N. Jindal, and A. Goldsmith, "Multi-antenna downlink channels 867 with limited feedback and user selection," IEEE J. Sel. Areas Commun., 868 vol. 25 , no. 7, pp. 1478-1491, Sep. 2007.

869 [25] K. K. Mukkavilli, A. Sabharwal, E. Erkip, and B. Aazhang, "On beam870 forming with finite rate feedback in multiple-antenna systems," IEEE 871 Trans. Inf. Theory, vol. 49, no. 10, pp. 2562-2579, Oct. 2003.

872 [26] G. Marsaglia, "Choosing a point from the surface of a sphere," Ann. Math. 873

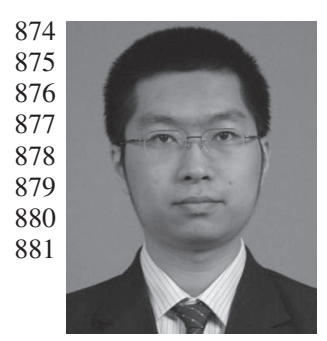

Yingxiang Yang received the B.E. degree from Tsinghua University, Beijing, China, in 2012. He is currently working toward the Ph.D. degree with Rutgers University, New Brunswick, NJ, USA.

His research interests include communications theory, optimization theory, and game theory.

Mr. Yang has been a Peer Reviewer for the IEEE Vehicular Technology Conference since 2011.

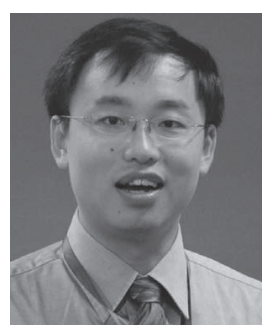

Wei Chen (SM'13) received the B.S. and Ph.D. 908 degrees in electronic engineering (first-class honors) 909 from Tsinghua University, Beijing, China, in 2002, 910 and 2007, respectively.

From 2005 to 2007, he was a Visiting Research 912 Staff with the Hong Kong University of Science and 913 Technology, Hong Kong. Since July 2007, he has 914 been with the Department of Electronic Engineer- 915 ing, Tsinghua University, where he is currently a 916 Full Professor and the Vice-Director of the Institute 917 of Communications. From June 2010 to September 918 2010, he was a Visiting Researcher with the University of Southampton, 919 Southampton, U.K. He is a 973 Youth Project Chief Scientist and is supported 920 by the New Century Talent Program of the Chinese Ministry of Education, 921 the Beijing Nova Program, and 100 fundamental research talents program of 922 Tsinghua University (also known as the 221 Talents Program). His research 923 interests include wireless communications, information theory, and applied 924 optimization.

925

Dr. Chen served as a Vice-Director of the Youth Committee of the China 926 Institute of Communications, a Tutorial Cochair of the 2013 IEEE International 927 Conference on Communications, a track Cochair of the Wireless Track of 928 the IEEE Consumer Communications and Networking Conference in 2013, a 929 Cochair of the Technical Program Committee (TPC) of the IEEE Vehicular 930 Technology Conference in Spring 2011, the Publication Chair of the IEEE 931 International Conference on Communications in China in 2012, a TPC Cochair 932 of the Wireless Communication Symposium at the 2010 IEEE International 933 Conference on Communications (ICC), and a Student Travel Grant Chair 934 of ICC 2008. He also served as an Editor for IEEE WiRELESS COMMU- 935 NICATIONS LETTERS. He received the 2010 IEEE Communication Society 936 Asia-Pacific Board Best Young Researcher Award; the 2009 IEEE Marconi 937 Prize Paper Award; the Best Paper Awards at the IEEE ICC in 2006, the 938 First IEEE International Workshop on Cross Layer Design in 2007, and the 939 IEEE Smart Gird Commmunications Conference in 2012; the 2011 Tsinghua 940 Rising Academic Star Award; the 2012 Tsinghua Young Faculty Teaching 941 Excellence Award; the First Prize at the First National Young Faculty Teach- 942 ing Competition; the First Prize at the Seventh Beijing Young Faculty Tea- 943 ching Competition; the First Prize at the Fifth Tsinghua University Young 944 Faculty Teaching Competition; and the National May 1st Labor Medal.

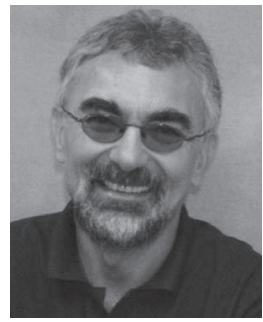

Lajos Hanzo (M'91-SM'92-F'04) received the 946 M.S. degree (with first-class honors) in electronics 947 and the Ph.D. degree from the Technical University 948 of Budapest, Budapest, Hungary, in 1976 and 1983, 949 respectively, the D.Sc. degree from the University of 950 Southampton, Southampton, U.K., in 2004, and the 951 "Doctor Honoris Causa" degree from the Technical 952 University of Budapest in 2009.

During his 35-year career in telecommunications, 954 he has held various research and academic posts in 955 Hungary, Germany, and the U.K. Since 1986, he has 956 been with the School of Electronics and Computer Science, University of 957 Southampton, Southampton, U.K., where he holds the Chair for Telecommuni- 958 cations. Since 2009, he has been a Chaired Professor with Tsinghua University, 959 Beijing China. He is currently directing a 100-strong academic research team, 960 working on a range of research projects in the field of wireless multimedia 961 communications sponsored by industry; the Engineering and Physical Sciences 962 Research Council, U.K.; the European IST Programme; and the Mobile Virtual 963 Centre of Excellence, U.K. He is an enthusiastic supporter of industrial and 964 academic liaison and offers a range of industrial courses. He has successfully 965 supervised $80 \mathrm{Ph} . D$. students, coauthored 20 John Wiley/IEEE Press books on 966 mobile radio communications totaling in excess of 10000 pages, published 967 more than 1250 research entries on IEEE Xplore, and presented keynote 968 lectures. (For further information on research in progress and associated 969 publications, please refer to http://www-mobile.ecs.soton.ac.uk.)

Dr. Hanzo is Fellow of the Royal Academy of Engineering, U.K., a Fellow 971 of the Institution of Electrical Engineers, and a Governor of the IEEE Vehicular 972 Technology Society. He has been a Technical Program Committee Chair and a 973 General Chair for IEEE conferences. During 2008-2012, he was the Editor-in- 974 Chief of the IEEE Press. He has received a number of distinctions.
906 Electronic Engineering Award from Tsinghua University and the Outstanding 907 Graduates of Shaanxi Province Award. 


\section{AUTHOR QUERIES}

AUTHOR PLEASE ANSWER ALL QUERIES

AQ1 = Please provide keywords

END OF ALL QUERIES 